Revue des patrimoines

Le patrimoine militaire et la question urbaine

\title{
Les enceintes de Strasbourg à travers les siècles
}

\section{Marie-Dominique Waton}

\section{OpenEdition}

Journals

Édition électronique

URL : http://journals.openedition.org/insitu/442

DOI : $10.4000 /$ insitu.442

ISSN : $1630-7305$

\section{Éditeur}

Ministère de la culture

\section{Référence électronique}

Marie-Dominique Waton, « Les enceintes de Strasbourg à travers les siècles », In Situ [En ligne],

16 | 2011, mis en ligne le 27 juin 2011, consulté le 30 avril 2019. URL : http://journals.openedition.org/ insitu/442 ; DOI : 10.4000/insitu.442

Ce document a été généré automatiquement le 30 avril 2019.

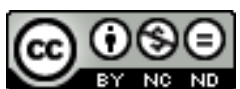

In Situ Revues des patrimoines est mis à disposition selon les termes de la licence Creative Commons Attribution - Pas d'Utilisation Commerciale - Pas de Modification 4.0 International. 


\title{
Les enceintes de Strasbourg à travers les siècles
}

\author{
Marie-Dominique Waton
}

1 Implantée au cœur des premières fortifications de la ville, la cathédrale de Strasbourg, joyau de la capitale alsacienne, voit progressivement croître la surface fortifiée. Depuis la chronique de Jacques Twinger de Koenigshoven (vers 1400), l'histoire des enceintes médiévales strasbourgeoises est présentée en termes «d'agrandissements » successifs depuis le noyau antique d'environ 20 ha: les deux premiers sont dus au pouvoir épiscopal, les deux suivants à la bourgeoisie. Devenue française en 1681, Strasbourg atteint les 275 ha d'espace fortifié avec Vauban et son ingénieur Tarade; les aménagements transforment la ville en forteresse, colonisée par des garnisons.

2 L'influence militaire a fortement marqué la trame urbaine de Strasbourg et cela, depuis ses origines: c'est ainsi qu'un certain nombre de constructions défensives - qu'elles soient antiques, médiévales, modernes voire contemporaines -, ne sont plus que perceptibles dans le tissu parcellaire actuel; d'autres font aujourd'hui partie de son patrimoine, par l'inscription ou par le classement au titre des Monuments historiques.

\section{Enceintes disparues mais visibles dans le tissu parcellaire}

3 C'est à la legio VIII Augusta, arrivée dans les années 90 de notre ère, que l'on doit l'essor d'Argentorate : c'est elle en effet qui est à l'origine de l'édification en dur, au cours du IIe siècle, d'une enceinte qui sera enchemisée au IVe siècle dans son extension définitive pour l'époque romaine.

4 La ville de Strasbourg a largement conservé l'empreinte du castrum et de la principale voie antique, que l'on peut remarquer dans la partie sud-est de l'ellipse insulaire. Au cours des siècles, le cardo maximus empruntera l'actuelle rue du Dôme ; la via quintana ${ }^{1}$, ancienne via principalis ${ }^{2}$, était située à peu près dans l'axe de l'actuelle petite rue du Tonnelet Rouge et se prolongeait vers le nord-ouest du côté sud-ouest de la rue de la 
Fonderie ${ }^{3}$ tandis que le tracé du decumanus maximus perdurera dans la rue des Hallebardes et une partie de la rue des Juifs. L'axe de sortie du camp, à partir de la rue de la Comédie et en direction du vicus de Koenigshoffen à l'ouest, est partiellement conservé dans le tissu parcellaire actuel, par la rue du Jeu des Enfants, longée sur son côté nord par la voie antique qui formait une sorte de chemin creux à son débouché occidental ${ }^{4}$. Cette voie empruntait ensuite la présente rue du Faubourg National.

(n) romaine semble avoir disparu, son trace en est encore partiellement visible dans le quart sud-est de l'ellipse insulaire (fig. $n^{\circ} 1$ ). Une trace de son angle ouest est encore perceptible dans l'angle nord-ouest de la place du Temple Neuf qu'il épouse. Sur le front sud-ouest du camp, la muraille est utilisée dans les fondations des immeubles, côté est de la place du Vieil-Hôpital ${ }^{5}$ tandis que, sur le front sud-est, la fortification qui passait au nord de la rue des Veaux, témoin parcellaire du fossé antique, se trouve vraisemblablement remployée dans le mur de la cave du n 23 .

\section{Figure 1}

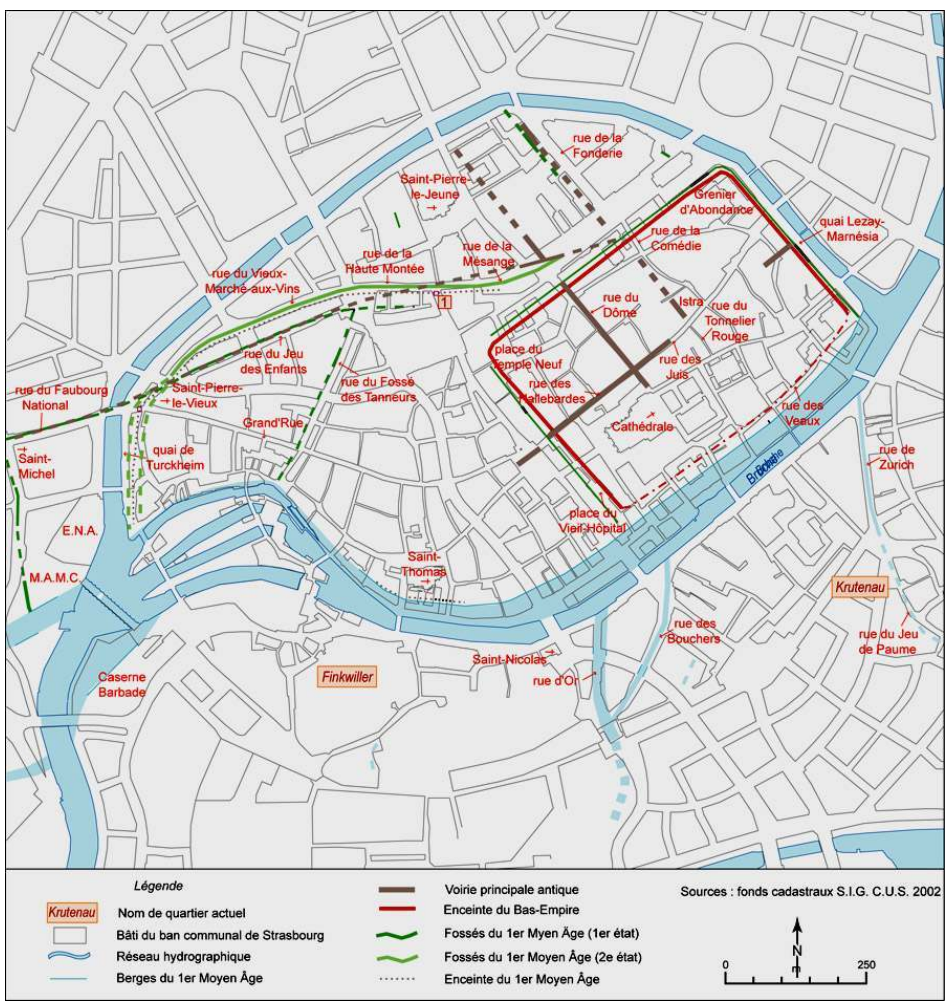

Strasbourg au Bas-Empire et au premier Moyen Âge. Phot. M.-D. Waton, 2010.

(c) DRAC Alsace, SRA.

6 Au cours du haut Moyen Âge, la ville, devenue Strateburgo - le bourg de la route - connaît le maintien du castrum, tandis que l'emprise de l'ellipse subit quelques transformations, perceptibles au travers d'un réseau de fossés (cf. fig. 1). Ainsi, un fossé alto-médiéval a précédé, à l'ouest de la rue du même nom ${ }^{6}$, celui du Fossé des Tanneurs. Des fossés des $\mathrm{X}^{\mathrm{e}}-\mathrm{XI}^{\mathrm{e}}$ siècles, perpétuant parfois des axes antiques, restent inscrits dans le parcellaire; localisés au nord de la rue du Jeu des Enfants, se prolongeant rue du Faubourg National puis bifurquant vers la rue de Molsheim, ils pourraient former limite de paroisses, comme par exemple celle de Sainte-Aurélie sur son côté est ${ }^{7}$. 
7 Le démantèlement du front sud-est de l'enceinte de l'Antiquité tardive est sans doute intervenu avant le XII ${ }^{e}$ siècle avec une transformation de l'espace fossoyé, dès le $\mathrm{IX}^{\mathrm{e}}-\mathrm{X}^{\mathrm{e}}$ siècle ${ }^{8}$, en espace de circulation conservé jusqu'à nos jours avec la rue des Veaux.

Pour la fin du premier Moyen Âge, deux pôles d'occupation ont été individualisés avec la ville "ancienne » (castrum) et une ville "neuve » d'environ 35 ha, délimitée par une enceinte, assurée au moins à partir de la rédaction du $1^{\mathrm{er}}$ statut municipal au cours du XII ${ }^{e}$ siècle ${ }^{9}$. Son tracé longerait, du côté sud, les rues de la Haute-Montée et du VieuxMarché-aux-Vins, lesquelles ont succédé au fossé retrouvé lors des fouilles de la ligne B du Tramway en 1998, pour redescendre vers la Bruche (actuellement l'ill), à l'ouest de l'église Saint-Pierre-le Vieux; le système défensif longerait ensuite, vers l'est, le cours d'eau en direction du Musée Historique. En-dehors de l'ellipse, quelques cours d'eau ont été repérés, ainsi ceux qui empruntaient les actuelles rues d'Or et des Bouchers, la rue de Zurich et, dans son prolongement vers le sud-est, la rue du Jeu de Paume, conditionnant le développement des enceintes ultérieures.

Jusque vers 1200 , la ville avait perpétué l'organisation antique avec l'enceinte du castrum et les églises paroissiales le long des axes principaux. Le XIII ${ }^{e}$ siècle marquera une rupture fondamentale par la réunion de ces ensembles avec la construction de l'enceinte dite épiscopale. Cette dernière donnera la forme qu'a conservé l'île dans sa partie septentrionale tandis qu'elle englobera une zone au sud de l'ill, en offrant une forme quasiment ovale (fig. $\mathbf{n}^{\circ} \mathbf{2}$ ). Le front nord-est du castrum, repris en sous-œuvre suite à la destruction d'une tour romaine, a été conservé et c'est de son angle nord-est que partait le tronçon nord de l'enceinte, d'une longueur de $1450 \mathrm{~m}$, pour rejoindre les actuels Ponts-Couverts. On peut découvrir le tracé du fossé, transformé en canal du FauxRempart dès 1228, en cheminant le long des quais Schoepflin, Kellermann, de Paris, Desaix et de Turckheim, sur tout le front nord de l'ellipse insulaire. Puis, depuis les PontsCouverts, le tronçon sud se développait sur une longueur de $1650 \mathrm{~m}$ pour rejoindre la rue de Zurich. Cette vaste enceinte englobait ainsi le castrum, la ville "neuve » et deux des anciennes paroisses périphériques, Saint-Pierre-le-Jeune au nord (17 ha) et Saint-Nicolas au sud (28 ha). 


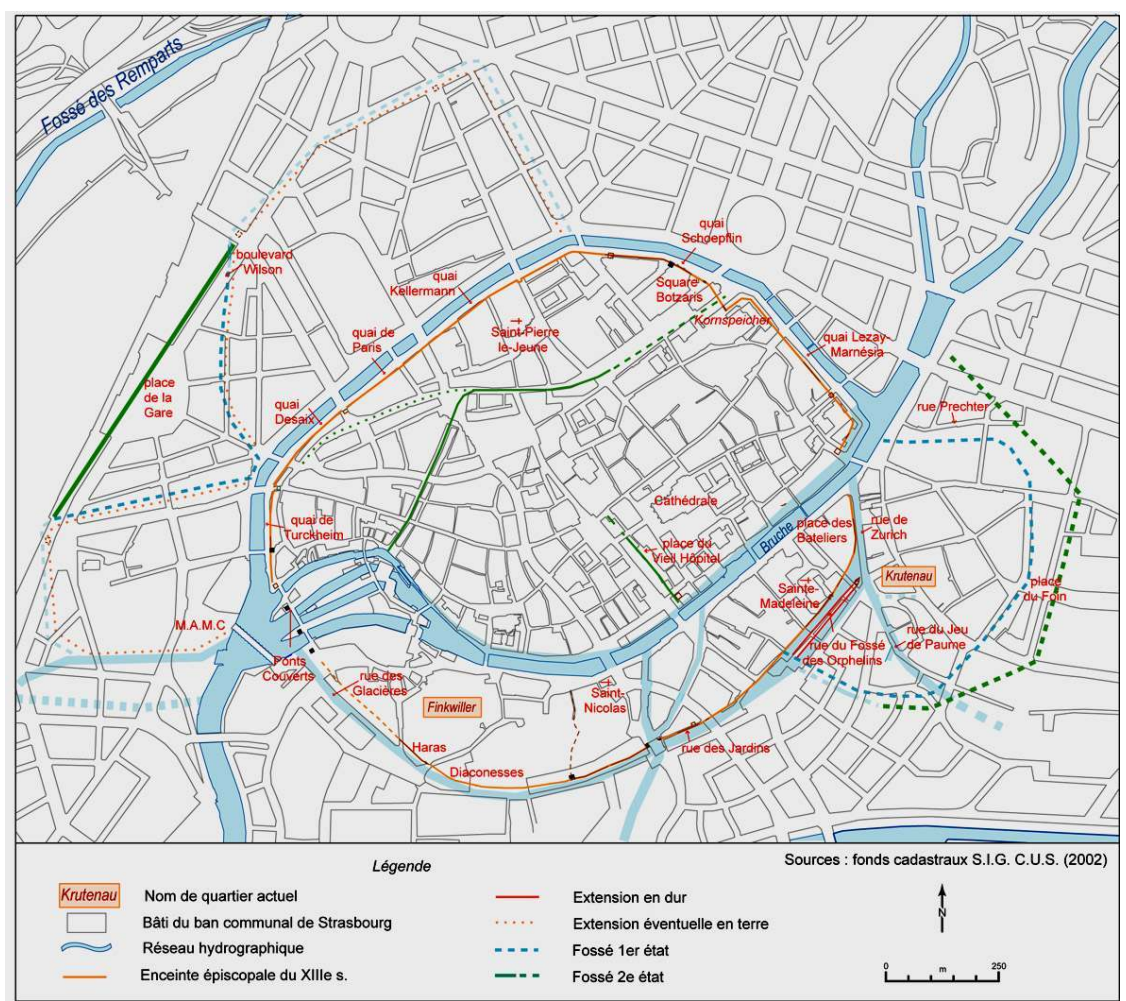

Strasbourg du XIIle siècle au troisième quart du XIVe siècle. Phot. M.-D. Waton, 2010.

(c) DRAC Alsace, SRA.

Si la partie ouest du tronçon sud a partiellement disparu jusqu'aux anciens Haras, où le mur défensif a été partiellement intégré dans l'habitat, on suit ensuite la fortification épiscopale dans l'actuel tissu urbain. Elle empruntait la rue des Jardins (fig. $\mathbf{n}^{\circ} \mathbf{3}$ ), longeait sur son côté nord la place des Orphelins et on la retrouve, encore en élévation, place Sainte-Madeleine; des travaux de réseaux ont permis de localiser l'enceinte basse en limite nord-ouest de la rue du Fossé des Orphelins. Puis, la muraille parcourait la rue de Zurich sur son côté ouest, cette rue matérialisant aujourd'hui l'ancien cours du Rheingiessen. 
Figure 3

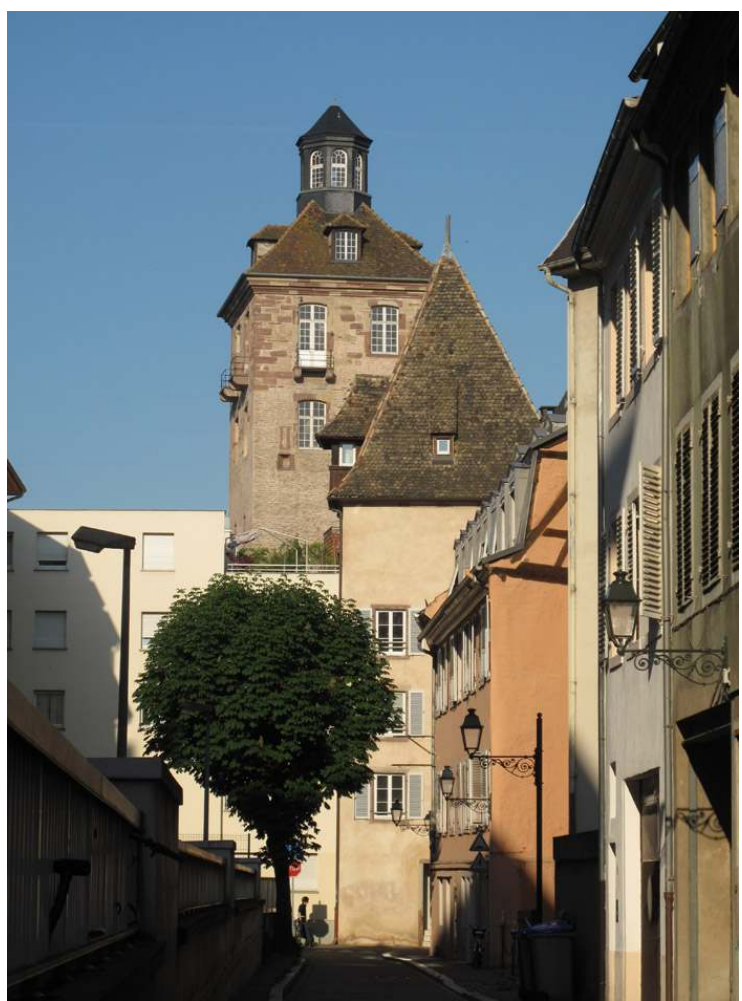

Vue de la tour pentagonale depuis la rue des Jardins ; en arrière-plan, le Spitalthor. Phot. M.-D. Waton, 2010.

(c) DRAC Alsace, SRA.

11 Dans la zone médiane de l'ellipse, un ancien fossé succédant aux fossés antiques et altomédiéval, initialement appelé fossé des Tailleurs puis Ulmergraber et faisant office d'égout, a été voûté avant 1424 ; son empreinte est encore ponctuellement visible au nord-ouest de la place du Vieil-Hôpital (fig. $\mathbf{n}^{\circ} 4$ ). 
Figure 4

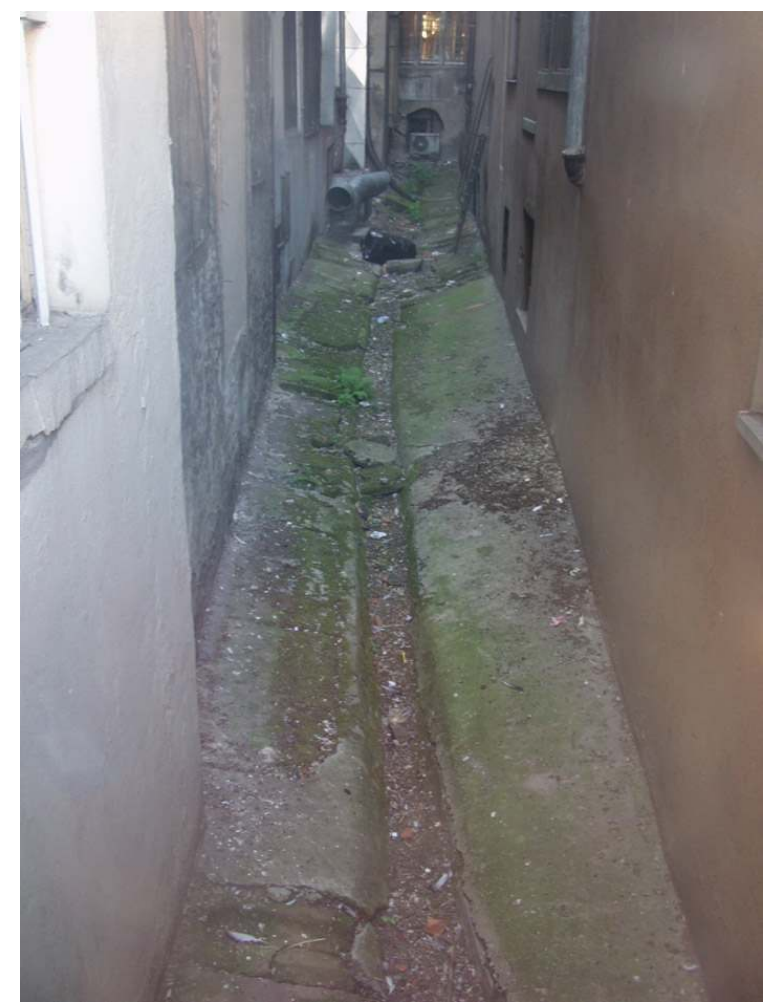

Témoin de l'Ulmergraben, place du Vieil-Hôpital. Phot. M.-D. Waton, 2003.

(c) DRAC Alsace, SRA.

Dans le faubourg ouest, plusieurs portes fortifiées sont mentionnées, impliquant l'existence probable de fossés et peut-être d'un rempart ou d'une palissade. Un fossé aura réuni les deux îlots, enclos de fossés. Les nombreuses observations, boulevard de Metz, sur la fortification du dernier tiers du XIVe siècle ( $c f$. infra) ont révélé une recharge de gravier en arrière de la muraille et, grâce à la fouille (inédite) effectuée boulevard Wilson ${ }^{10}$, on peut émettre l'hypothèse que le mur a été installé dans le creux formé par le " nouveau fossé » ( $c f$ f fig. 2 où celui-ci est figuré en vert), réalisé antérieurement ${ }^{11}$ (fig. $\mathbf{n}^{\circ}$ 5). Ces vestiges sont en retrait des boulevards qui matérialisent une aire de circulation évoquée plus loin. 
Figure 5

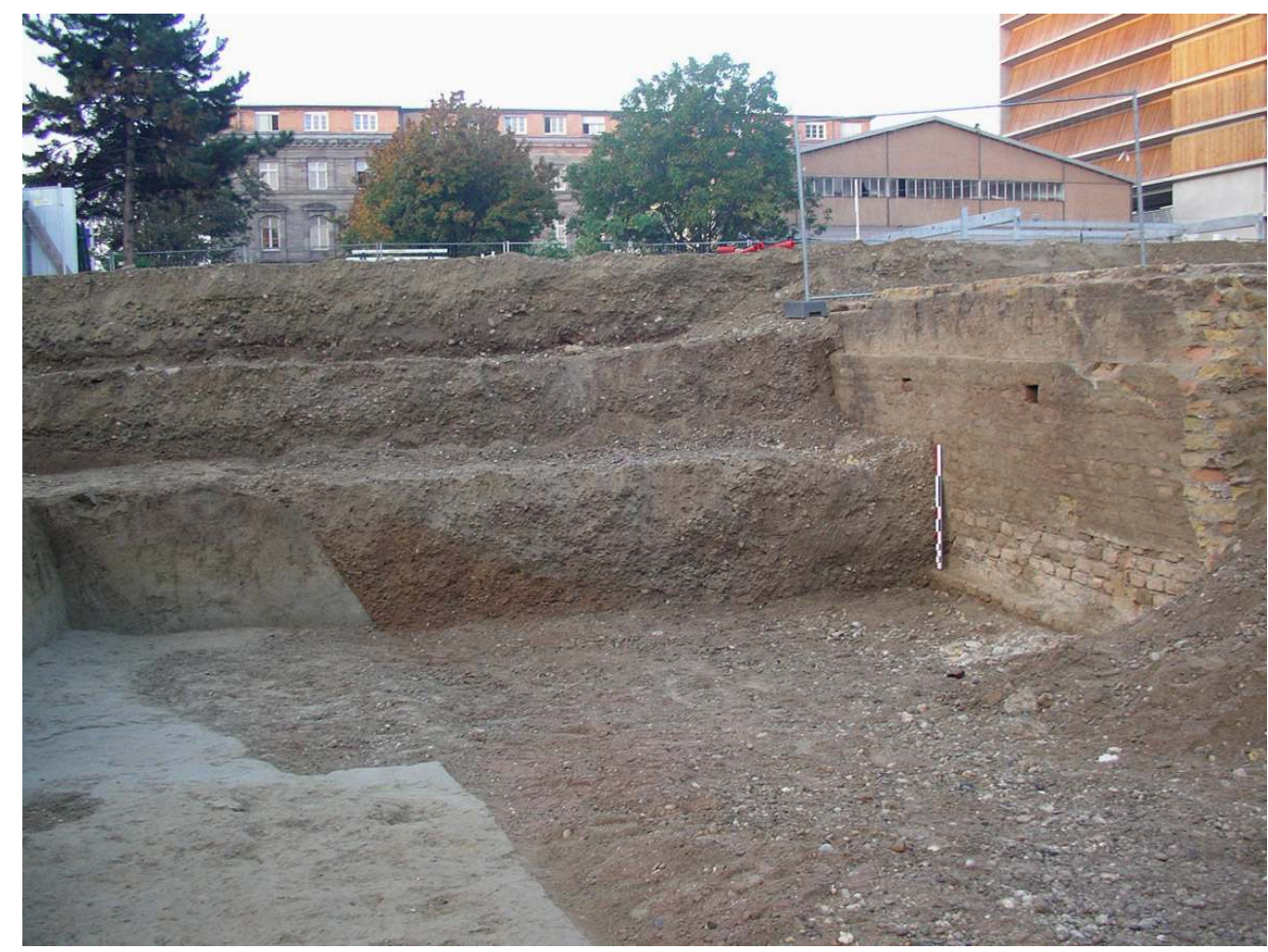

Coupe stratigraphique, à l'arrière de l'enceinte du XIVe siècle, montrant l'empreinte d'un fossé sur le chantier du boulevard Wilson. Phot. Yves Henigfeld, 2007.

(c) INRAP, Grand-Est Sud.

Le faubourg de la Krutenau a sans doute connu un processus de défense légère ${ }^{12}$ dont le tracé hypothétique prend en compte le parcellaire actuel et quelques observations ponctuelles. Au nord, en retrait de la rue Prechter ${ }^{13}$ ancienne "rue Neuve», une dépression a été observée et à l'ouest, place du Foin, des informations anciennes signalent également une profonde dépression: on propose de reconnaître dans les rues des Balayeurs et de l'Abreuvoir, se développant respectivement au nord et au sud de la place du Foin, les indices parcellaires du système de défense originel de la Krutenau au début du second Moyen Âge.

14 Au cours de la seconde moitié du XIV ${ }^{\mathrm{e}}$ siècle, la ville entreprend d'agrandir son périmètre fortifié par des enceintes maçonnées; ce sera d'abord le faubourg ouest (76 ha) avec le «troisième agrandissement » puis, en rive droite de l'ill, le faubourg est ( 27 ha) dès le début du $\mathrm{XV}^{\mathrm{e}}$ siècle avec le "quatrième agrandissement ». La superficie fortifiée atteint alors 202 ha (fig. $\mathbf{n}^{\circ} \mathbf{6}$ ). 


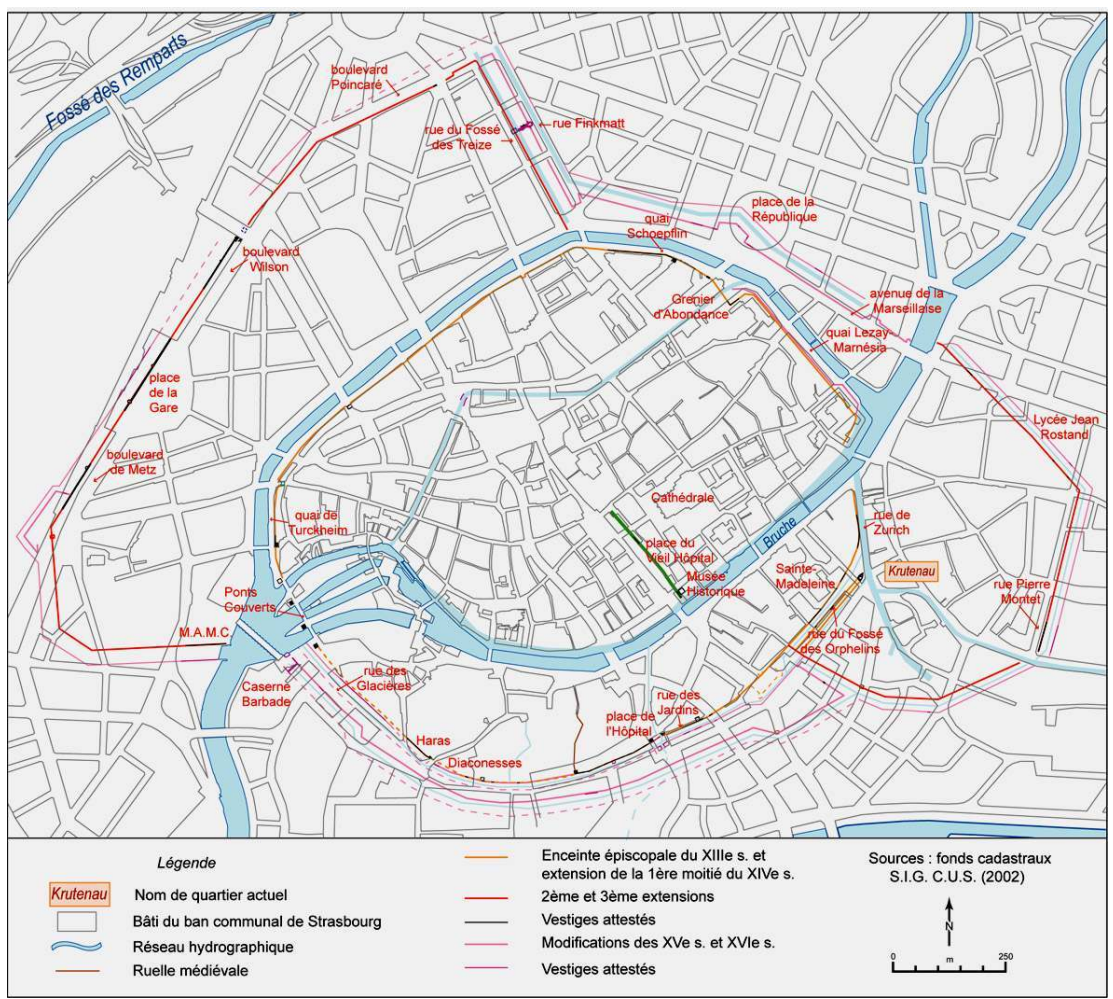

Strasbourg dans le dernier quart du XIVe siècle et au XVe siècle. Phot. M.-D. Waton, 2010 (c) DRAC Alsace, SRA.

Le faubourg occidental est enclos par une muraille longue de près de $2600 \mathrm{~m}$; partant de l'Ill au sud des bâtiments de l'ENA, elle longe les boulevards de Metz, traverse la place de la Gare où la contrescarpe se trouve sous le mur de façade de la gare, longe ensuite le boulevard Wilson, emprunte la rue du Président Poincaré et, après un angle droit, vient rejoindre le canal du Faux-Rempart en longeant la rue du Fossé des Treize du côté est. Quant au faubourg de la Krutenau, clé du Rhin et qui a été fortifié par un mur sur près de $1000 \mathrm{~m}$, on reconnaît le tracé de la muraille dans la rue Pierre Montet qu'elle bordait du côté sud ${ }^{14}$.

Les fouilles rue des Glacières, aux Haras et aux Diaconesses ont montré des reprises importantes, remarquables notamment dans le changement de module des briques employées ; la rue des Glacières reste le témoin de la trace du fossé, situé entre la faussebraie et l'enceinte, reconstruite à la place d'une contrescarpe et reconnue sur près de $70 \mathrm{~m}$ de long lors de fouilles préventives au $\mathrm{n}^{\circ} 9$ de la rue ${ }^{15}$.

La rue Finkmatt est l'un des rares témoins parcellaires du XVI siècle, gardant la trace du fossé dédoublé avec la création de la fausse-braie nord-est, terminée par Daniel Specklin, lequel avait initié une réorganisation complète du système fortifié; celle-ci sera entreprise dès 1633 d'après un plan-masse établi par Paul Mörschauser, général de l'armée suédoise, et terminée par Christophe Heer, architecte de la ville jusqu'à l'arrivée des Français.

Sur les fronts sud et sud-est, si l'enceinte médiévale est maintenue, la fausse-braie est détruite pour laisser place à un système bastionné (fig. $\left.\mathbf{n}^{\circ} \mathbf{7}\right)$. L'empreinte d'un des quatre bastions réalisés perdure dans la forme d'un bâtiment érigé au sein des Hospices civils. 
Par ailleurs, on a pu constater qu'une grande partie de ces constructions avait pris appui sur les murs de contrescarpe de la Krutenau et sur le mur de la fausse-braie qui n'avait été qu'arasé tandis que, pour le front ouest, la nouvelle fortification avait remployé le mur d'escarpe médiéval. Ce fut alors qu'un talus avait été installé à l'arrière du mur et que l'espace de circulation s'était ainsi trouvé éloigné de l'enceinte, explication de l'indice parcellaire révélé par les boulevards Wilson et de Metz, à distance de la muraille.

Figure 7

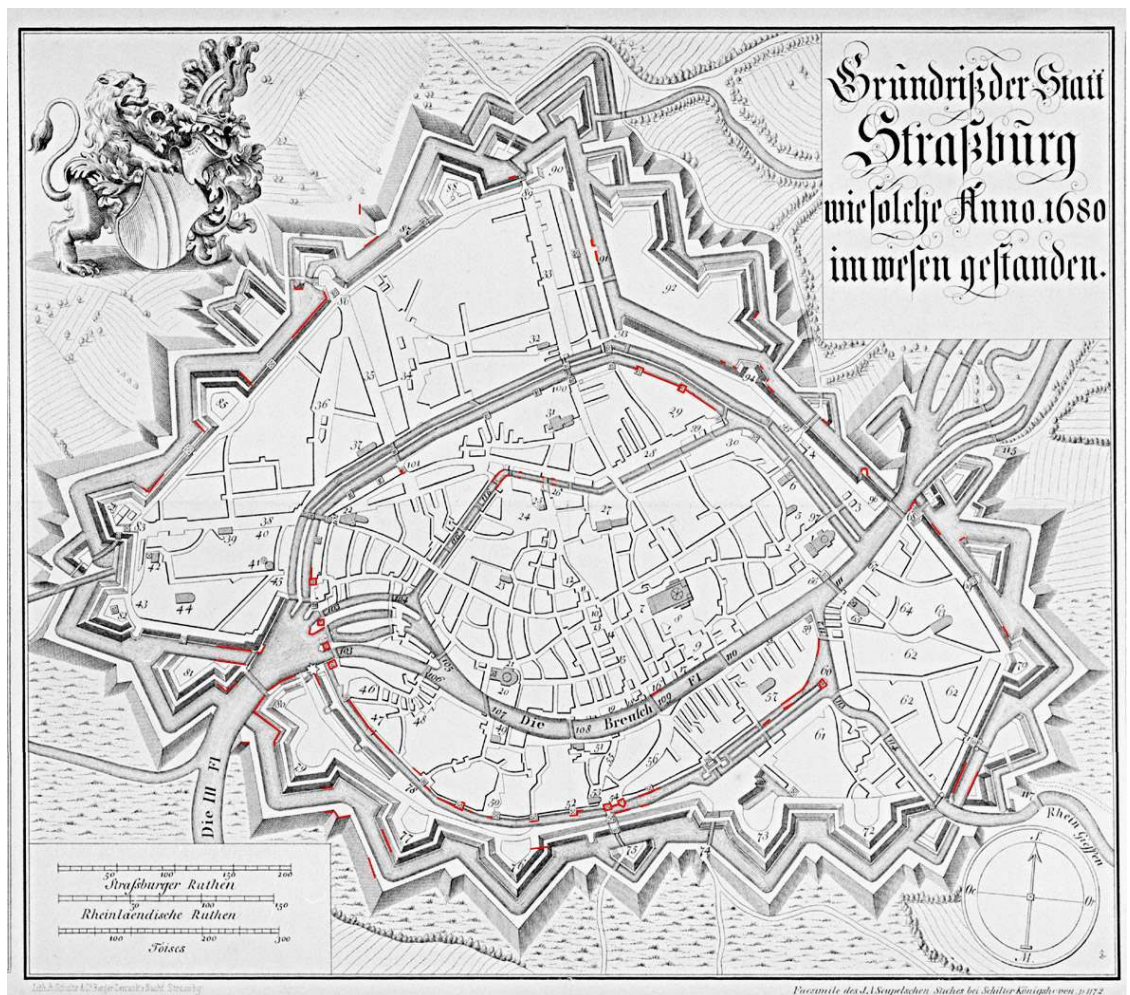

Plan de Schoepflin (copie de Seupel) avec localisation des vestiges reconnus par les archéologues (C DRAC Alsace, CRMH, ICO482D001_001_nuc2, Schultz et Berger-Levrault ; Phot. M.-D. Waton, 2010.

(c) DRAC Alsace, SRA.

D'autre part, la modification de l'enceinte à l'extrémité est de l'ellipse insulaire, qui n'apparaît pas sur le plan de Schoepflin mais que révèle le plan-relief de $1725 / 27$, est perceptible dans le tissu parcellaire actuel à l'extrémité sud du quai Lezay-Marnésia.

\section{Éléments défensifs conservés avec protection Monument historique ${ }^{16}$}

Les Allemands sont les premiers à s'être intéressés aux vestiges antiques avec la conservation au début du $\mathrm{XX}^{\mathrm{e}}$ siècle, sur le front sud-ouest du castrum, d'une tour du BasEmpire $^{17}$; cette dernière est visible dans les sous-sols d'un magasin, 47-49 rue des Grandes Arcades.

Sur le front nord-ouest du camp, le mur d'enceinte antique sert encore de fondation au mur sud du Grenier d'Abondance ${ }^{18}$, aujourd'hui annexe de l'Opéra du Rhin (place du Petit Broglie); on peut apercevoir l'enceinte du Haut-Empire à chaînages de terres cuites 
architecturales (briques et/ou tegulae) contre l'hôtel du Gouverneur, à l'ouest du Grenier d'abondance, où a été, dans les années 2000, ré-ensevelie une tour du Bas-Empire, observée par le facteur d'orgues Jean-André Silberman, en 1765.

Quant à l'enceinte épiscopale, un tronçon de la partie sud est parfaitement conservé place Sainte-Madeleine où un portail, daté de 1576 et provenant d'un hôtel de la rue Brûlée démoli en 1913, forme passage entre la place et la rue du Fossé des Orphelins. Cette portion, pourvue ultérieurement d'un chemin de ronde et de merlons très larges (fig. $\mathbf{n}^{\circ}$ 8), est inscrite sur la liste des monuments protégés ${ }^{19}$.

Figure 8

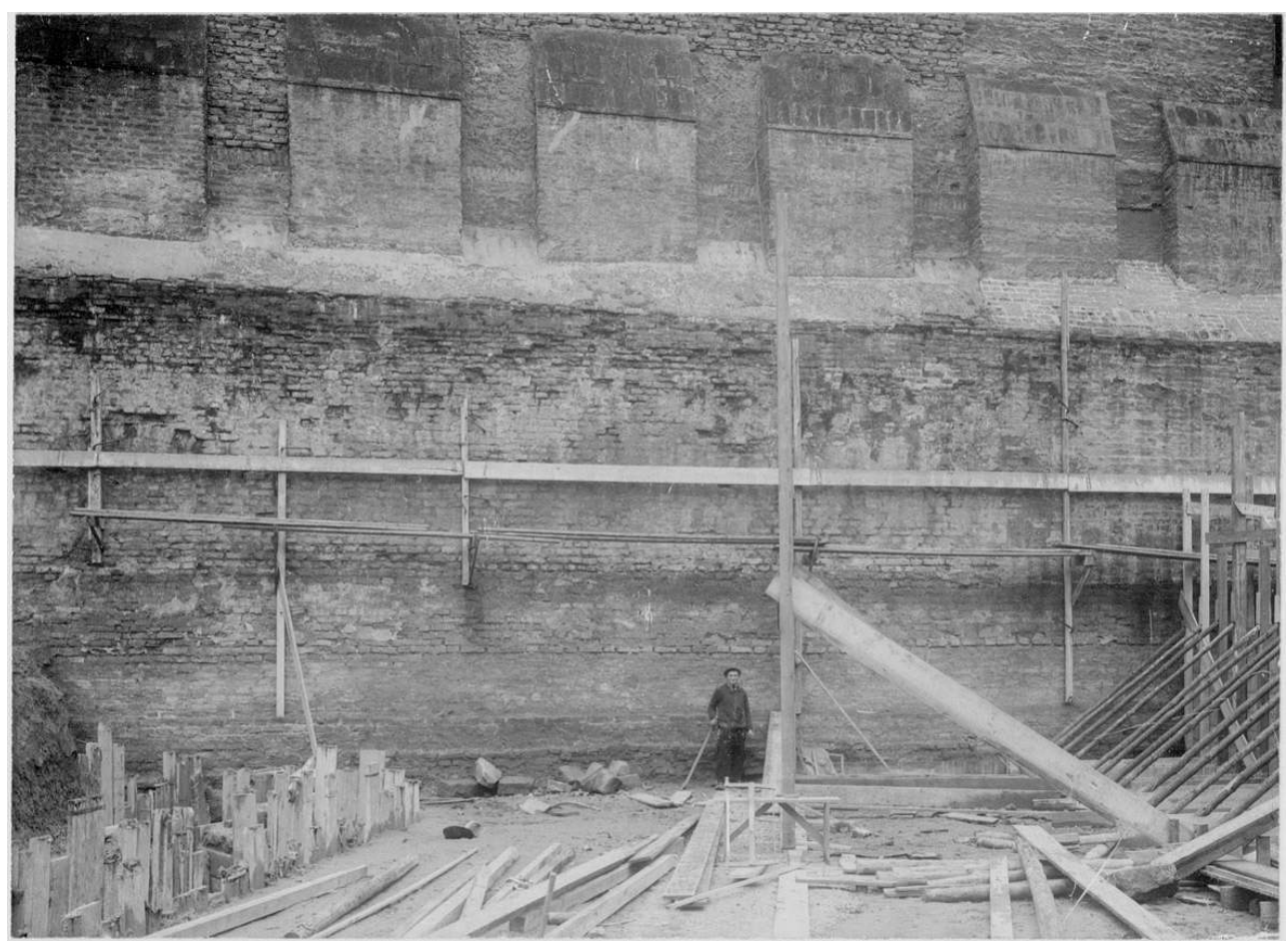

Vue de la courtine, en cours de restauration, place Sainte-Madeleine.

(c) DRAC Alsace, CRMH, ICO482B183_002, Jules Manias, 1909.

Deux tours-portes sont conservées en élévation; l'ancienne porte dite Bündethor, encore visible dans l'enceinte des Hospices civils (fig. $\mathbf{n}^{\circ} \mathbf{9}$ ), avait été rétrogradée au profit du Spitalthor, situé à $172 \mathrm{~m}$ environ vers l'est. La façade du Bündethor tournée vers la campagne est intégrée dans le bâtiment qui remplaça l'hôpital, disparu dans un incendie en 1716. Ce bâtiment moderne, qui infléchit son plan selon la courbure de l'enceinte médiévale, a été classé le 13 juin 1926. 
Figure 9

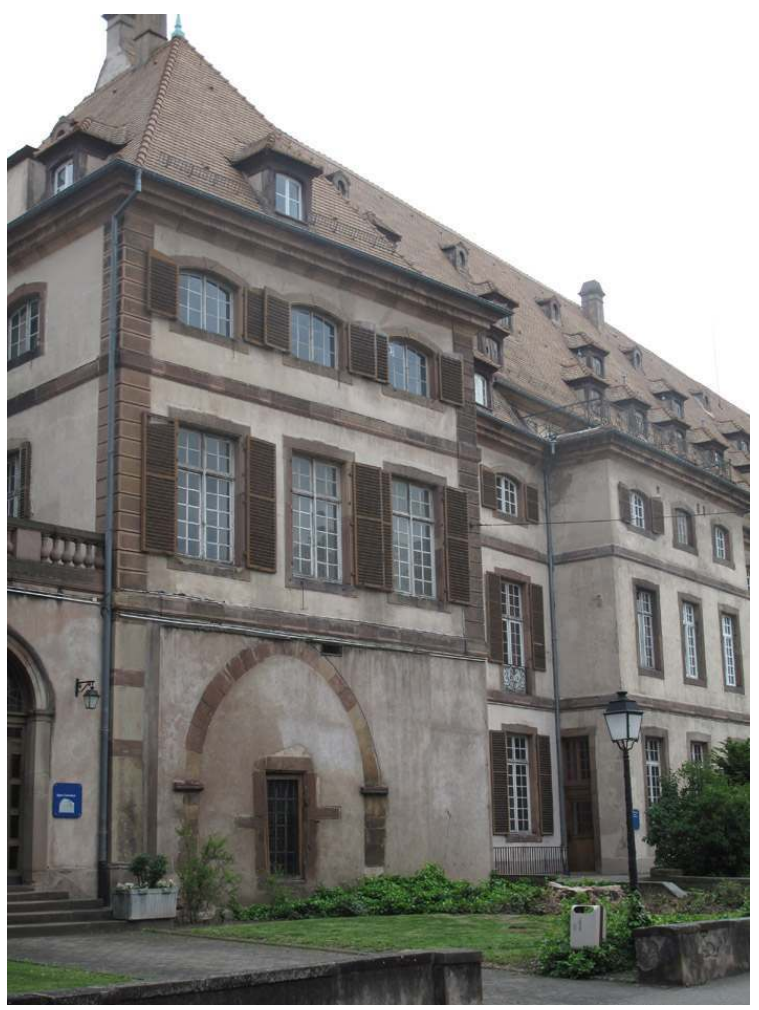

Le Bünderthor, muré en 1932, au sein des Hospices civils. Phot. M.-D. Waton, 2010.

(c) DRAC Alsace, SRA.

24 La porte de l'Hôpital ou Spitalthor, également protégée ${ }^{20}$, a été observée sur le plan archéologique avec une analyse des élévations par M. Werlé lors de son étude de la tour pentagonale voisine $\mathrm{e}^{21}$ (fig. $\mathbf{n}^{\mathbf{0}} \mathbf{1 0}$ ), également inscrite au titre des Monuments historiques (protection incluse dans celle de la porte de l'Hôpital). Le Spitalthor, de plan quadrangulaire massé $(8,80 \times 8,90 \mathrm{~m})$, s'élevait sur cinq niveaux dans son état primitif et, d'après quelques indices techniques de datation (typologie des pierres à bossage, profil des moulures...), il semblerait que la tour-porte puisse être datée de la première moitié du XIV siècle plutôt que de la fin de ce siècle. 


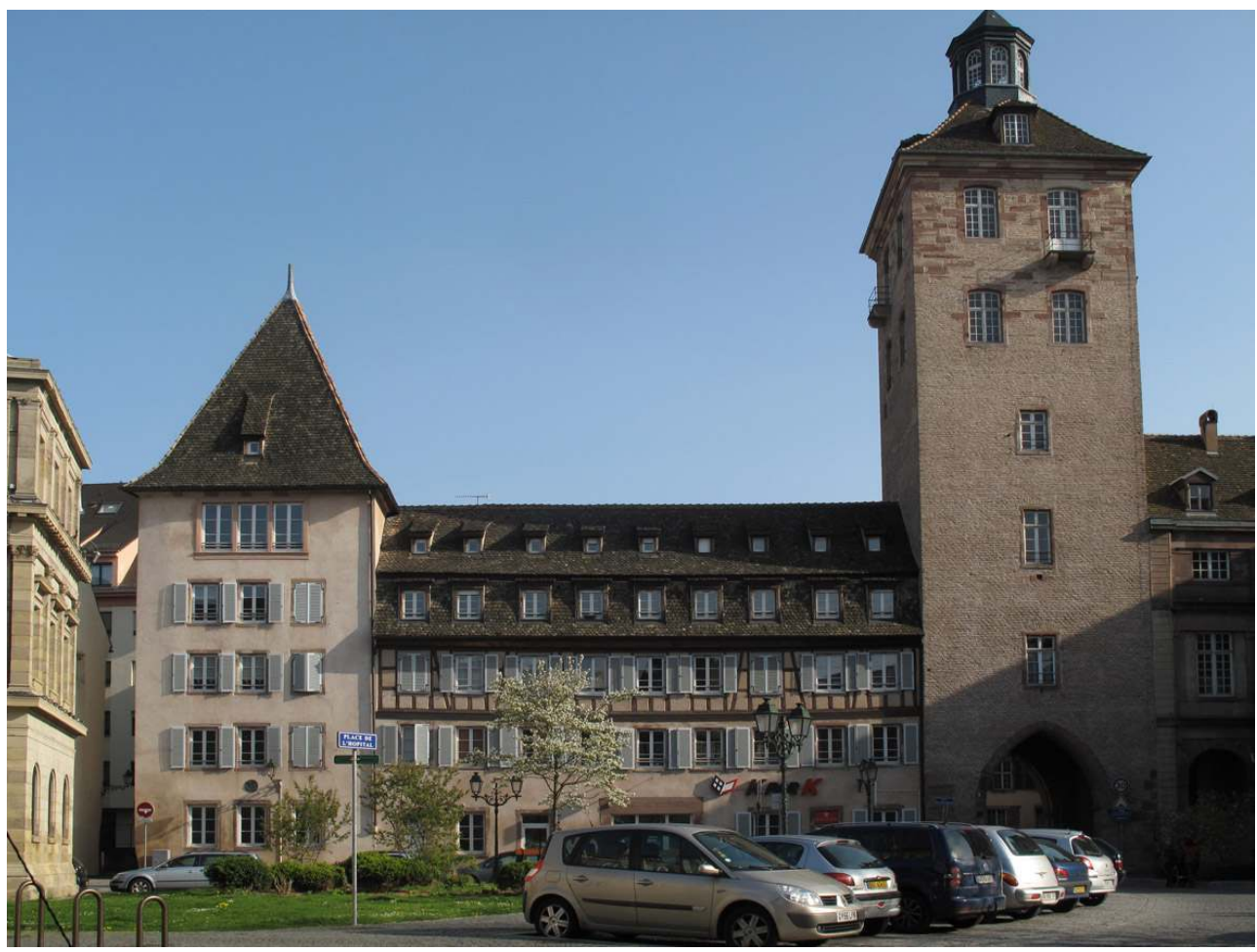

Place de l'Hôpital avec, à gauche, la tour pentagonale et, à droite, la tour-porte de l'Hôpital. Phot. M.-D. Waton, 2010.

(c) DRAC Alsace, SRA.

Unicum au sein du système défensif mis en œuvre, l'étude archéologique des élévations de la tour pentagonale, sise au 9/10 place de l'Hôpital, a montré que le plan de cet ouvrage était "fortuit », hérité d'un état daté du deuxième quart du XIII ${ }^{\mathrm{e}}$ siècle, correspondant à la mise en défense de l'entrée d'un chenal dans la ville ${ }^{22}$, lequel desservait les fossés d'Or ( Goldgiessen) et des Bouchers (Metzgergiessen). Cet ouvrage primitif, formant un angle à pan coupé pourvu d'une meurtrière, servit de soubassement à une tour édifiée autour des années 1300 et qui, ouverte à la gorge, s'élevait sur une hauteur observée de 15,60 m audessus du sol actuel. Vers 1491, la tour a été couronnée d'une toiture haute de 9,60 m, dotée d'une échauguette et couverte de tuiles creuses. 
Figure 11

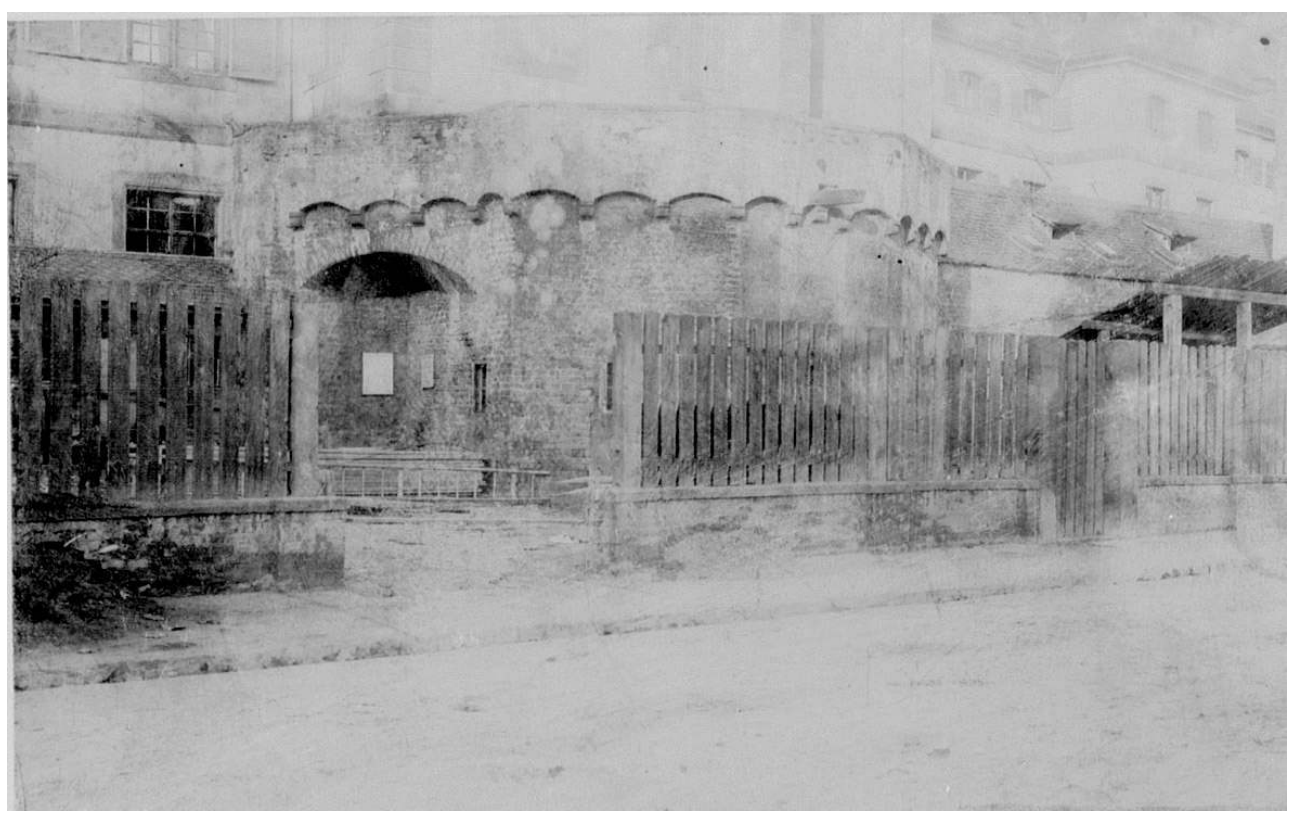

Vue du bastion pentagonal dans l'enceinte des Hospices civils.

(c) DRAC Alsace, CRMH, ICO482B104_009, 1903.

Dans l'enclos des Hospices civils, on peut voir encore, masqué aujourd'hui sous de la vigne vierge, un bastion polygonal (fig. $\mathbf{n}^{\circ} \mathbf{1 1}$ ), inclus dans la protection de 1929.

Sur le tronçon nord qui ceinture encore partiellement l'ellipse insulaire, ce sont les bâtiments de l'ancienne fonderie de canons, reposant sur une portion de l'enceinte et incluant la tour médiévale côté quai (fig. $\mathbf{n}^{\circ} \mathbf{1 2}$ ), qui ont fait l'objet d'une protection au titre des Monuments historiques le 12 octobre $1929^{23}$. La tour garde le témoin de l'arrachage du mur d'enceinte à sa base actuelle. 
Figure 12

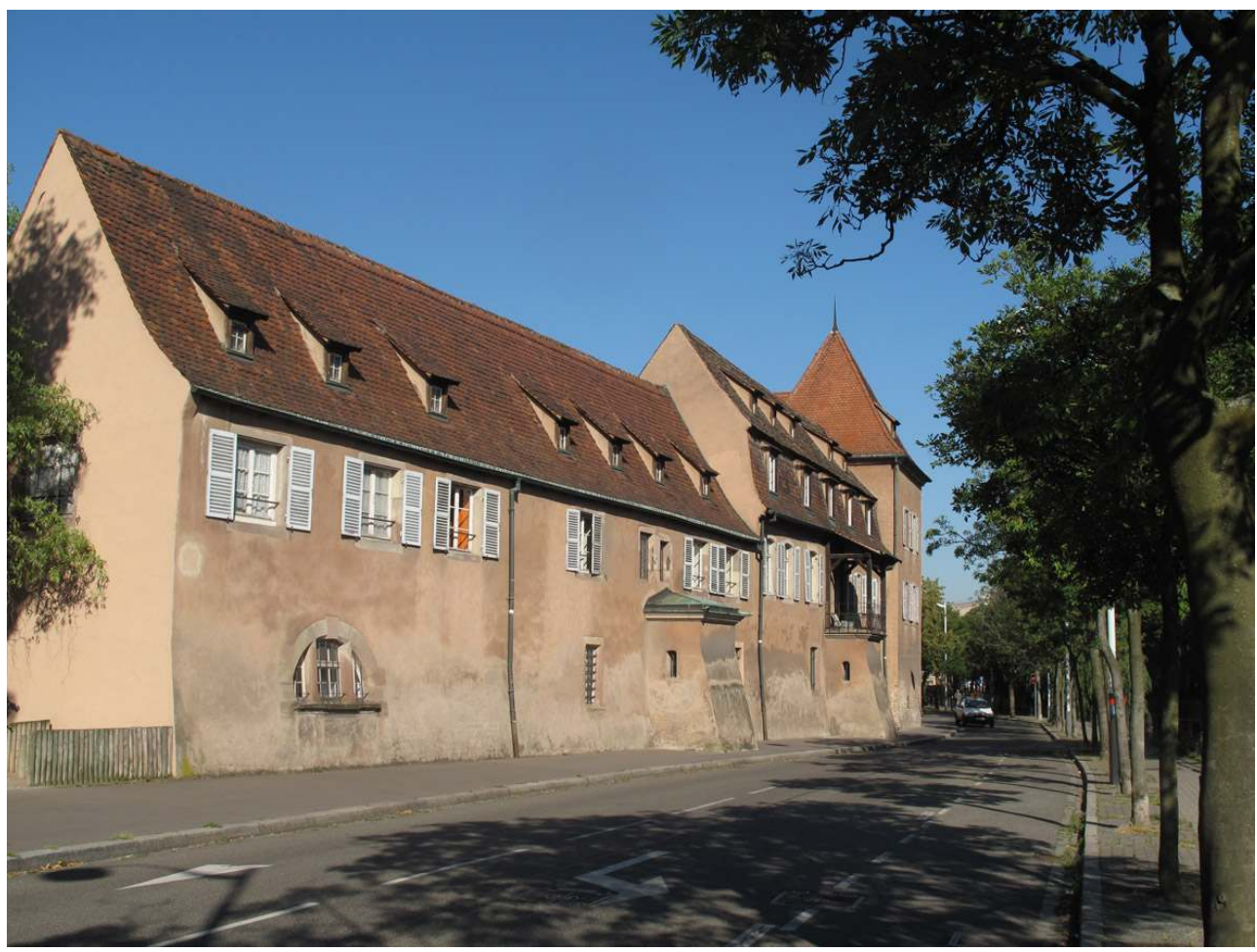

Vue de l'enceinte avec une tour médiévale, quai Schoepflin. Phot. M.-D. Waton, 2010.

(c) DRAC Alsace, SRA.

28 Quai de Turckheim, la tour a vu sa protection incluse dans l'arrêté de classement du 6 juillet 1928, paru au J.O. du 16 février 1930, portant sur les « quatre tours » sises près des Ponts-Couverts ${ }^{24}$.

Figure 13

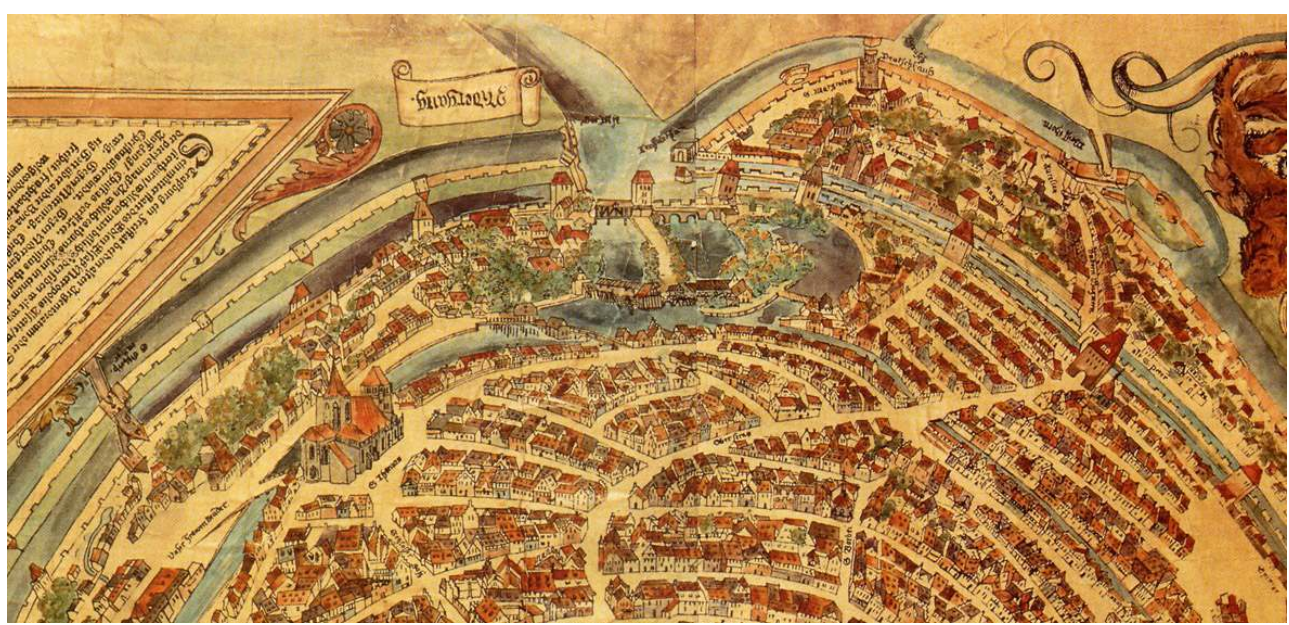

Extrait du plan C. Morant (1548), d'après le plan dépl. h.-t. publié par Liliane Châtelet-Lange. Strasbourg en 1548 : le plan de Conrad Morant. Strasbourg : Presses universitaires de Strasbourg, 2001. Phot. Yves Henigfeld, 2008.

(c) INRAP, Grand-Est Sud. 
La configuration occidentale de l'ellipse insulaire où la Bruche se divisait en trois canaux en 1548 (fig. $\mathbf{n}^{\circ} \mathbf{1 3}$ ) a nécessité un traitement défensif adapté. Des quatre tours, mentionnées pour la première fois en 1300, subsistent la Heinrichsturm, la Hans von Altheimturm et la tour des Français ${ }^{25}$. La première, au nord, la Maltzenturm, a été rasée en 1869, suite à un incendie (fig. $\mathbf{n}^{\circ} \mathbf{1 4}$ ). Les Ponts-Couverts, dont l'appellation (Bedeckte Brücken) avait été justifiée par la succession de galeries couvertes d'une toiture reliant les tours les unes aux autres, détruites en 1784, resteront la ligne de défense extrême de la ville jusqu'au dernier quart du XVIIe siècle.

Figure 14

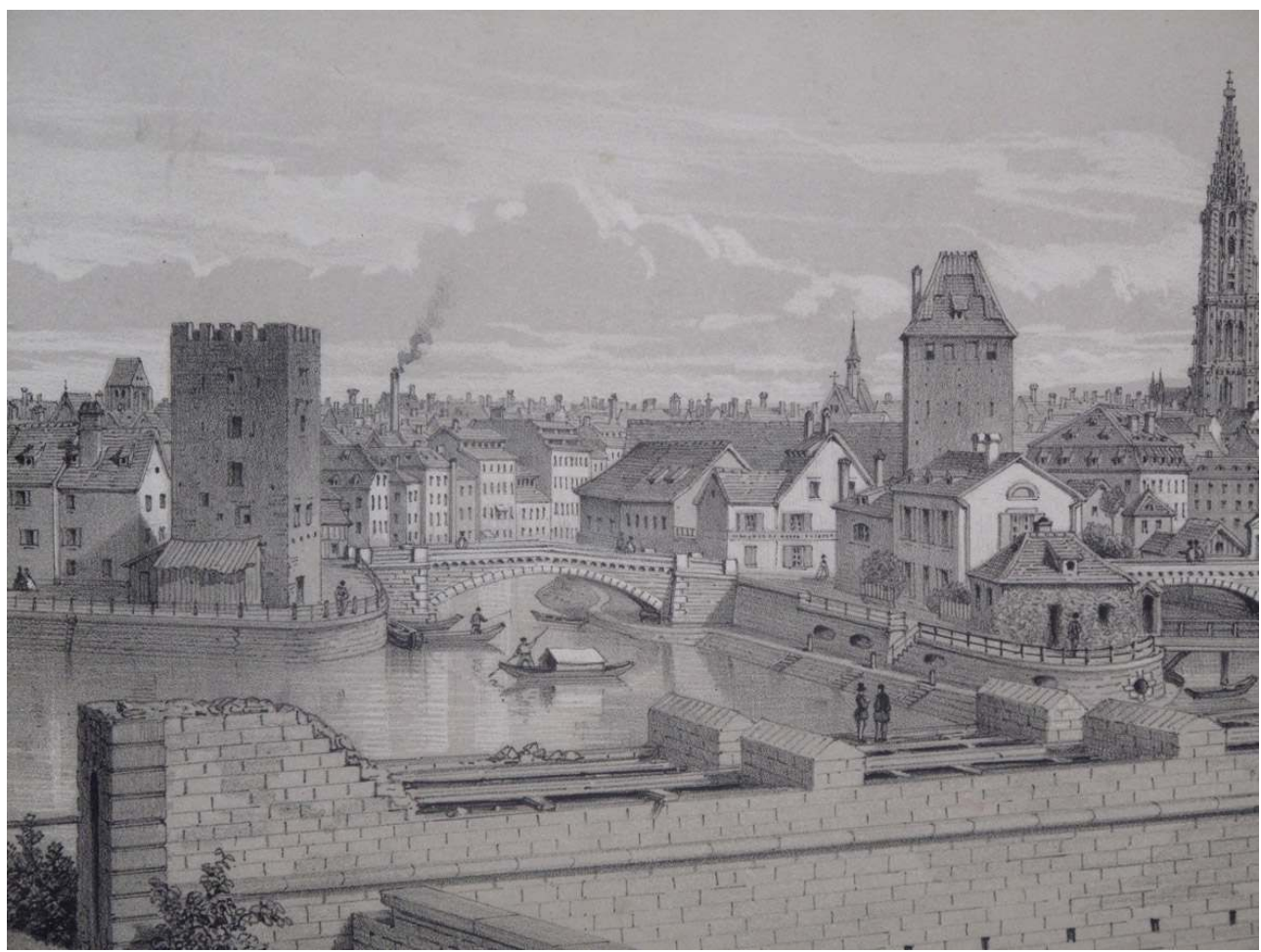

Extrait d'une lithographie, tirée vers 1900, représentant les Ponts-Couverts dessinés par A. Maugembre vers 1869 : coll. privée. Phot. M.-D. Waton, 2010.

(c) DRAC Alsace, SRA.

30 Ainsi, de l'ensemble médiéval fortifié, il subsiste hors du sol les trois tours dites des Ponts-Couverts qui, initialement crénelées ont, par la suite été coiffées d'une toiture réalisée en tuiles creuses (fig. $\mathbf{n}^{\circ} \mathbf{1 5}$ ), une tour quai Turckheim et une tour quai Schoepflin, avec une portion de courtine; sont également sauvegardés la tour pentagonale de la place de l'Hôpital, deux tours-portes, deux tronçons de fortification, l'un dans l'enclos de l'Hôpital Civil, l'autre place Sainte-Madeleine. La majorité de ces éléments sont protégés au titre des Monuments historiques 


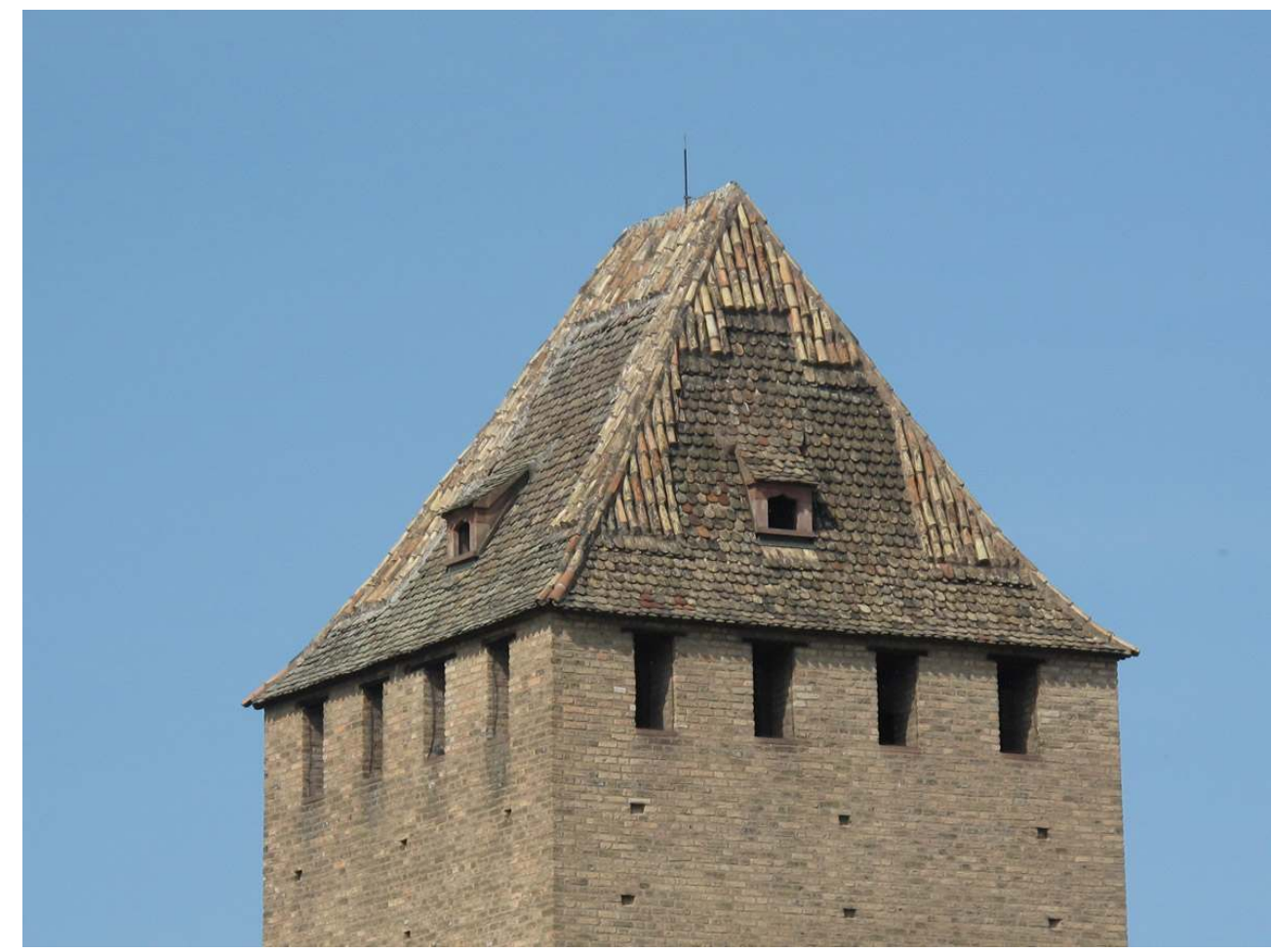

Détail de la toiture de la Heinrichsturm. Phot. M.-D. Waton, 2010.

(c) DRAC Alsace, SRA.

$31 \mathrm{Du}$ "troisième agrandissement ", la «tour du Diable ", dite aussi "bastion SaintMaximilien» et dont la protection est incluse dans celle du barrage Vauban, est conservée à proximité du Musée d'art moderne et contemporain et les vestiges de l'enceinte réutilisée dans le système bastionné, repérés sur près de $140 \mathrm{~m}$ boulevard Wilson ${ }^{26}$ (fig. $\mathbf{n}^{\circ} \mathbf{1 6}$ ), ont été protégés au titre des Monuments historiques ${ }^{27}$ le 29 octobre 2001. 


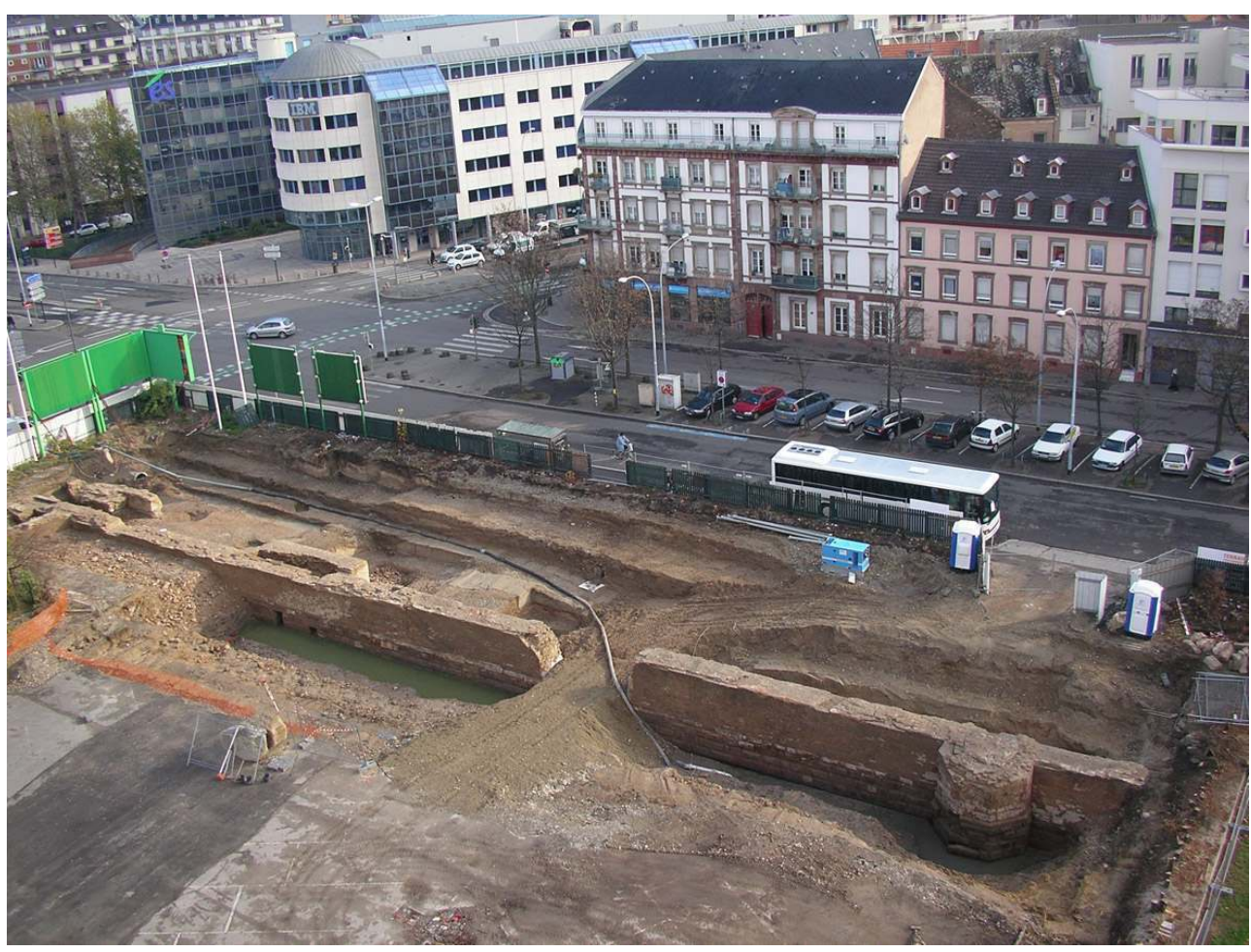

Boulevard Wilson, vue générale des vestiges. Phot. Y. Henigfeld, 2007.

(c) INRAP, Grand-Est Sud.

Parmi les tours de guet, une seule tour est conservée en élévation et protégée au titre des Monuments historiques depuis le 30 décembre 1985; il s'agit de la tour dite Breuscheck, attestée en 1392, 33 rue de la Tour à Koenigshoffen ${ }^{28}$.

Avec le XVIe siècle et Specklin apparaîtront les premiers plans de Strasbourg actuellement conservés : plans de Conrad Morant (1548), d'Abraham Hoggenberg (1572), de Daniel Specklin ${ }^{29}$ repris au XIX ${ }^{\text {e }}$ siècle..., qui nous permettent d'avoir une vision globale de la ville. Ensuite, il faut citer ici comme sources d'étude le précieux plan-relief de la ville daté de 1725/1727, remarquablement présenté au Musée historique (fig. $\mathbf{n}^{\circ}{ }^{17}$ ), et le plan parcellaire de 1765 relevé par l'architecte J.-F. Blondel. Celui-ci conserve de nombreux aspects de l'organisation topographique de la fin du Moyen Âge. Au XIX ${ }^{\mathrm{e}}$ siècle, un premier plan cadastral est réalisé en 1837 : N.-J. Villot et F. Piton retranscriront dans le détail le tracé des fortifications Vauban. Parallèlement, un second plan-relief, actuellement au Musée des Invalides à Paris, a été exécuté en 1836 et remis à jour jusqu'en $1863^{30}$. 


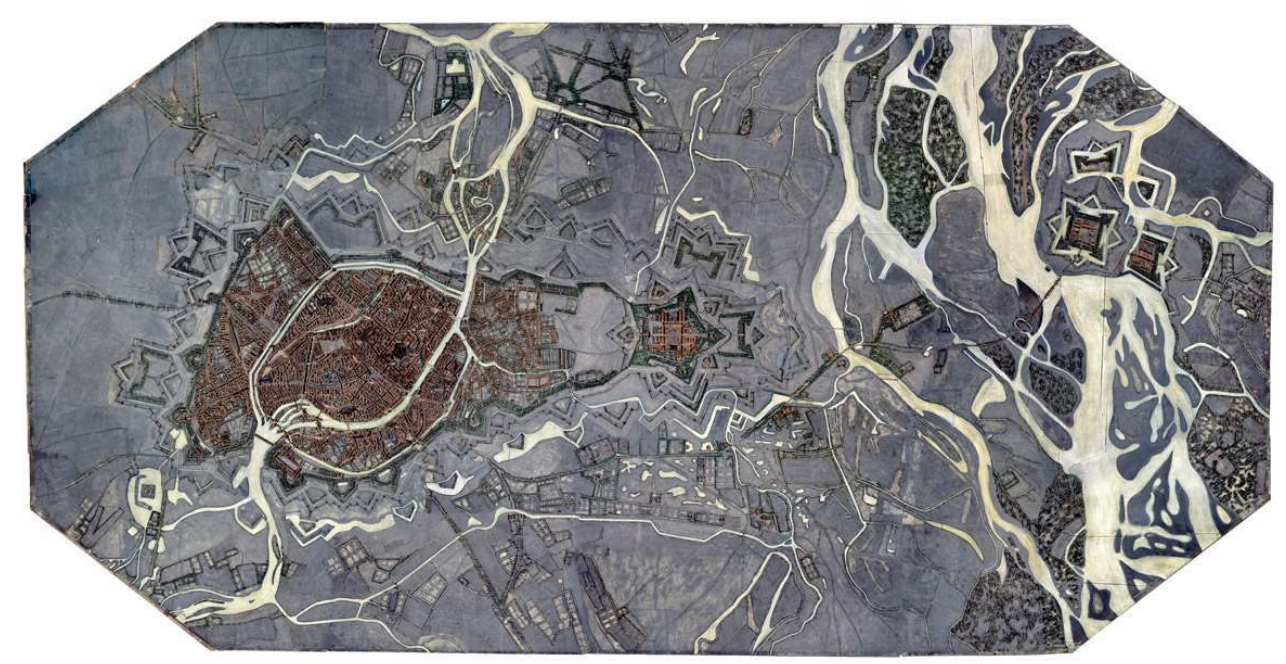

Plan-relief de 1725/27, conservé au Musée Historique de Strasbourg. Phot. Claude Menninger.

(C) Région Alsace - Inventaire général. comme en témoigne le plan-relief de 1725/27, remplacé superficiellement par un système de vannes d'eau à la fin du XVIII ${ }^{e}$ - début du XIX ${ }^{e}$ siècle, avec cinq portes tournant autour d'un axe à peu près centré, et dynamité au XIX ${ }^{e}$ siècle pour la construction des Abattoirs, a été déplacé pour la construction du Musée d'art moderne et contemporain (fig. $\mathbf{n}^{\circ} \mathbf{1 8}$ ). Sa protection, en date du 7 décembre 1995, a été incluse dans celle de la "grande écluse " ${ }^{32}$, édictée le 18 mai 1971. Débutée en 1686, la construction de cette dernière, également dénommée barrage Vauban, a été conçue pour inonder les terrains situés au sud de la ville, en fermant les vannes afin de faire monter le niveau de l'Ill. Appelé également « pont Vauban » ou " passage Vauban », le barrage contient un couloir menant d'une rive à l'autre avec trois ponts-levis, aménagé par G. Frankhauser au début du XX siècle. 
Figure 18

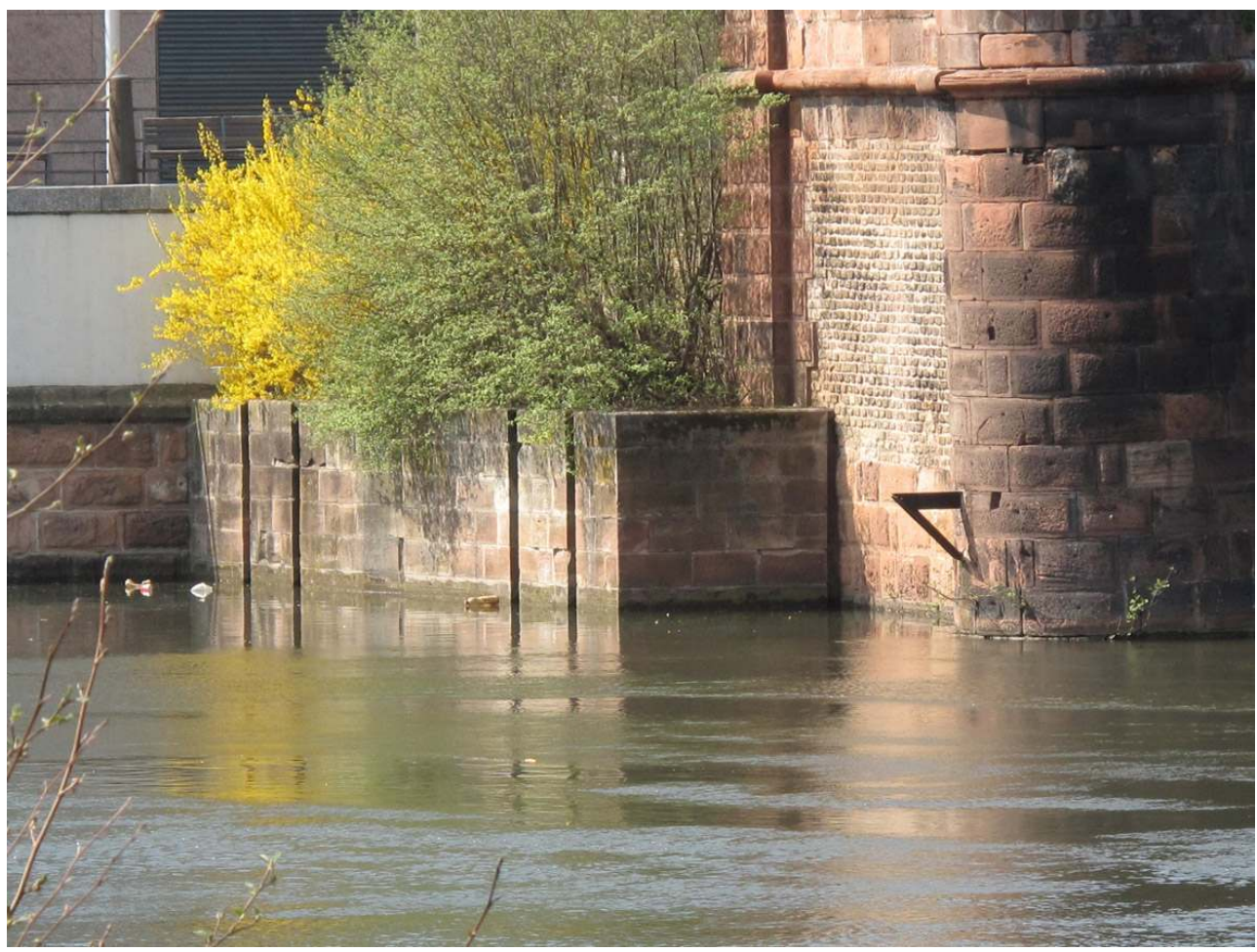

Élément de vannes d'eau mises au jour sur le site du futur Musée d'art moderne et contemporain, déplacé à côté de la « tour du Diable » (à droite) et pierres de l'enceinte du XIVe siècle en remploi (à gauche). Phot. M.-D. Waton, 2010.

(c) DRAC Alsace, SRA.

36 Face à l'Allemagne, la citadelle de forme pentagonale avec un bastion à chaque angle (fig. $\left.\mathbf{n}^{\circ} 19\right)$ était reliée à la ville par deux courtines bastionnées. Très fortement endommagée par les bombardements de 1870, elle a été partiellement démontée dès 1896 ; il n'en reste qu'une demi-lune avec la courtine entre deux bastions, dans le parc de la Citadelle, correspondant à la porte d'accès côté Rhin et qui a été classée les 27 avril 1922 et 14 octobre $1932^{33}$. 


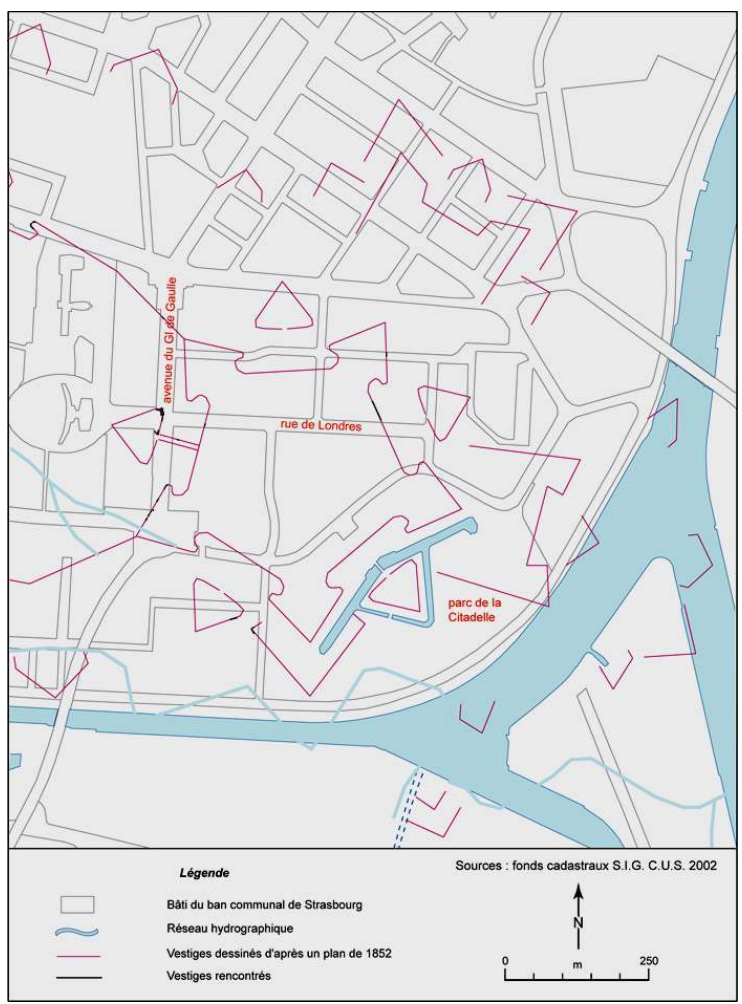

Plan de la Citadelle de Strasbourg à la fin du XVIle siècle. Phot. M.-D. Waton, 2010.

(c) DRAC Alsace, SRA.

Le siège de la ville en 1870 ayant révélé la faiblesse de son système défensif, les Allemands entreprirent de renforcer ce dernier après la guerre (fig. $\mathbf{n}^{\circ} \mathbf{2 0}$ ). Les anciennes fortifications furent remplacées par une enceinte constituée d'une succession de bastions, dominés par des cavaliers et aux flancs très courts, et de courtines sur un plan polygonal simplifié. Le rempart, précédé d'un fossé en eau, était destiné à abriter des casernes à l'abri des bombes, des magasins à poudre et des locaux d'artillerie. L'enceinte de Strasbourg (Stadumwallung) était alors, avec celle de Cologne, la plus importante des enceintes urbaines édifiées en Allemagne ${ }^{34}$. Ces fortifications allemandes, sur leurs fronts nord et nord-est, ont été inscrites ${ }^{35}$ par arrêté du 2 avril 2009. 


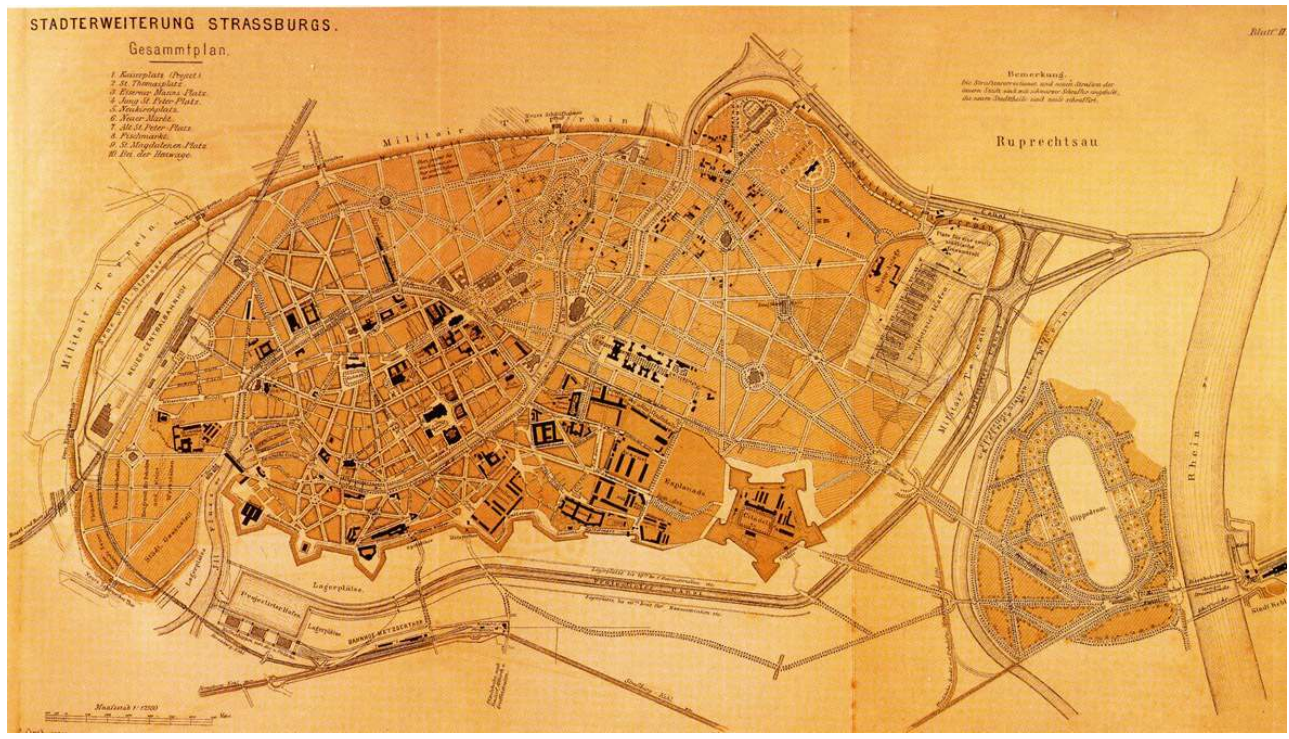

Fortifications allemandes et projet d'urbanisation de la ville en 1878. Phot. Yves Henigfeld, 2008.

(c) INRAP, Grand-Est Sud.

\section{Éléments défensifs conservés sans protection}

C'est encore aux Allemands que l'on doit la conservation d'une portion de la muraille avec chaînage de terres cuites architecturales du Haut-Empire (fig. $\mathbf{n}^{\circ} \mathbf{2 1}$ ), dans une crypte aménagée sous les jardins de la Préfecture, à l'est du Grenier d'Abondance, et réalisée pendant la dernière guerre; on peut également y voir le mur du Bas-Empire avec l'amorce d'une tour ainsi que sa jonction avec l'enceinte épiscopale. Le tout avait été dégagé par F. Garscha en $1941^{36}$. 
Figure 21

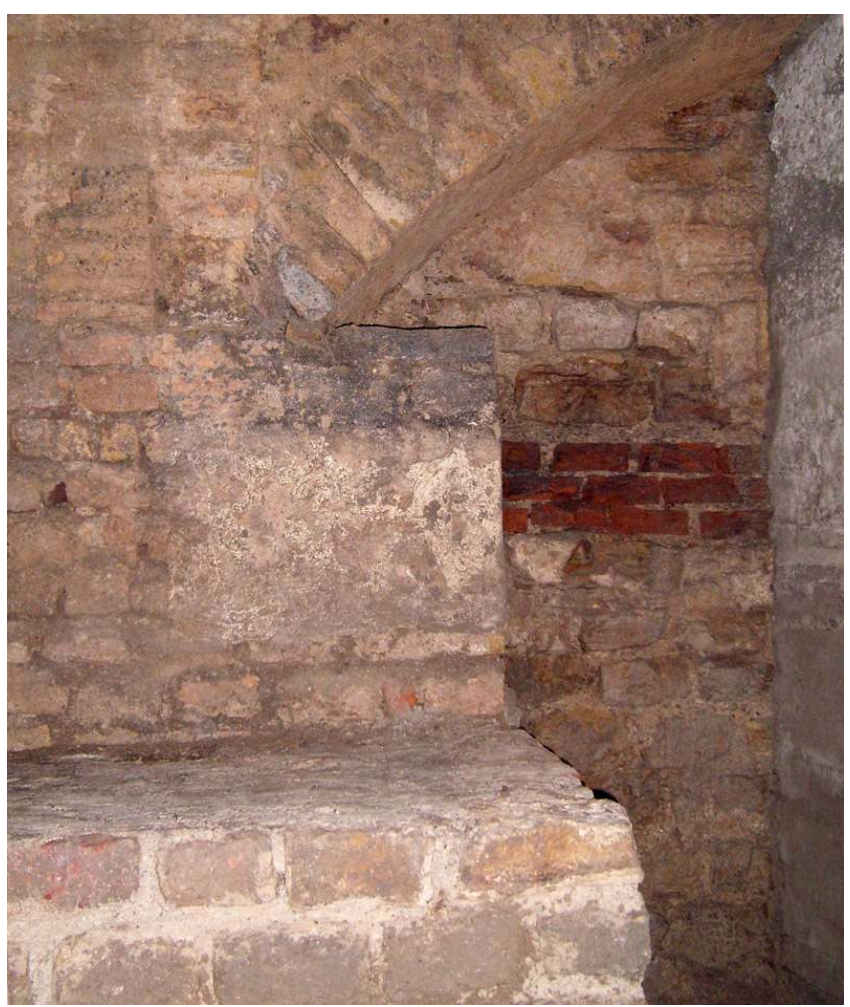

Dans la crypte des jardins de la préfecture, parmi les fondations du Grenier d'Abondance, l'enceinte du Haut-Empire en arrière-plan et, en avant-plan, l'enceinte du Bas-Empire. Phot. Dominique Bonneterre, 2008.

(c) DRAC Alsace, SRA.

Les vestiges du système fortifié du «troisième agrandissement », localisés boulevard de Met $^{37}$, sont conservés dans le sous-sol strasbourgeois après que la disposition des pieux ait été modifiée dans les nouvelles constructions et adaptée pour leur sauvegarde dans le sol. Une tourelle (Schneke) de plan polygonal, mise au jour place de Gare ${ }^{38}$, a été remontée chemin des Glacis (fig. $\mathbf{n}^{\circ} \mathbf{2 2}$ ). 


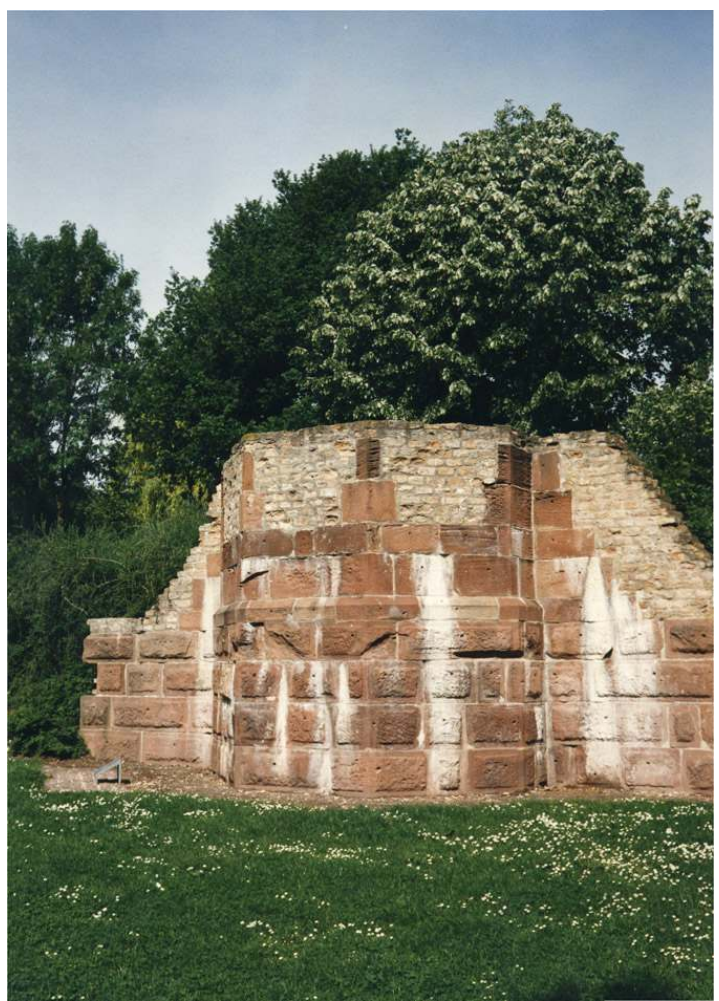

Tourelle du XIVe siècle, découverte place de la Gare et remontée chemin du Glacis. Phot. Guy Feuerbach, 2004.

(c) DRAC Alsace, SRA.

40 Si aucun vestige de l'enceinte de la Krutenau dite du « quatrième agrandissement » n'a été protégé, une poterne inconnue des textes est néanmoins conservée dans un bâtiment universitaire (L'Escarpe), rue Pierre Montet. À l'est, sur la berge du quai du Maire Dietrich où la fortification aboutissait contre la rivière au niveau de la porte des Pêcheurs, on peut encore voir des éléments de quai en pierres à bossage, terminés par une tour également construite avec des pierres à bossage, en partie basse et en chaînage d'angles, et une élévation de briques jaunes (fig. $\left.\mathbf{n}^{\circ} \mathbf{2 3}\right)$. 
Figure 23

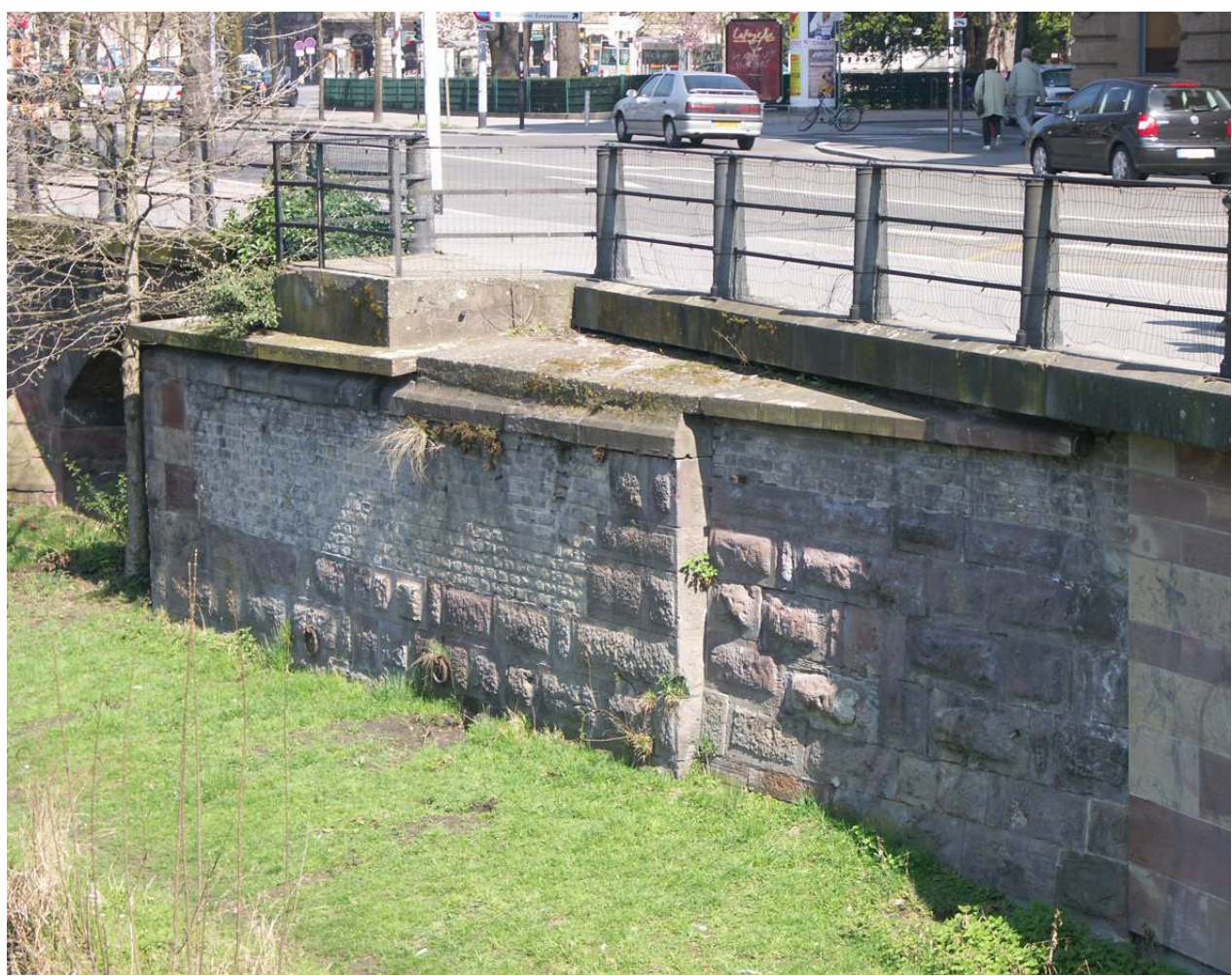

Quai du Maire Dietrich, tour et quai du début du XVe siècle. Phot. M.-D. Waton, 2005.

(c) DRAC Alsace, SRA.

Des vestiges bastionnés, érigés au XVIIe siècle et dont l'essentiel existera jusqu'au début du XXe siècle, seul un tronçon est visible dans le parking de l'Hôtel du Département, construit à la place de la Caserne Barbade; la pierre de fondation du «bastion des Païens » de 1630 a été retrouvée lors des fouilles de la place de la Gare.

Vauban avait également étendu le front des fortifications par la construction de nombreuses demi-lunes dont une fouillée au sud, dans l'enceinte de l'Hôpital civil ${ }^{39}$. L'échauguette de cette dernière, arasée au début $\mathrm{du} \mathrm{XX}^{\mathrm{e}}$ siècle, avait été déplacée par les

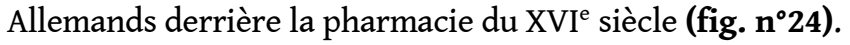




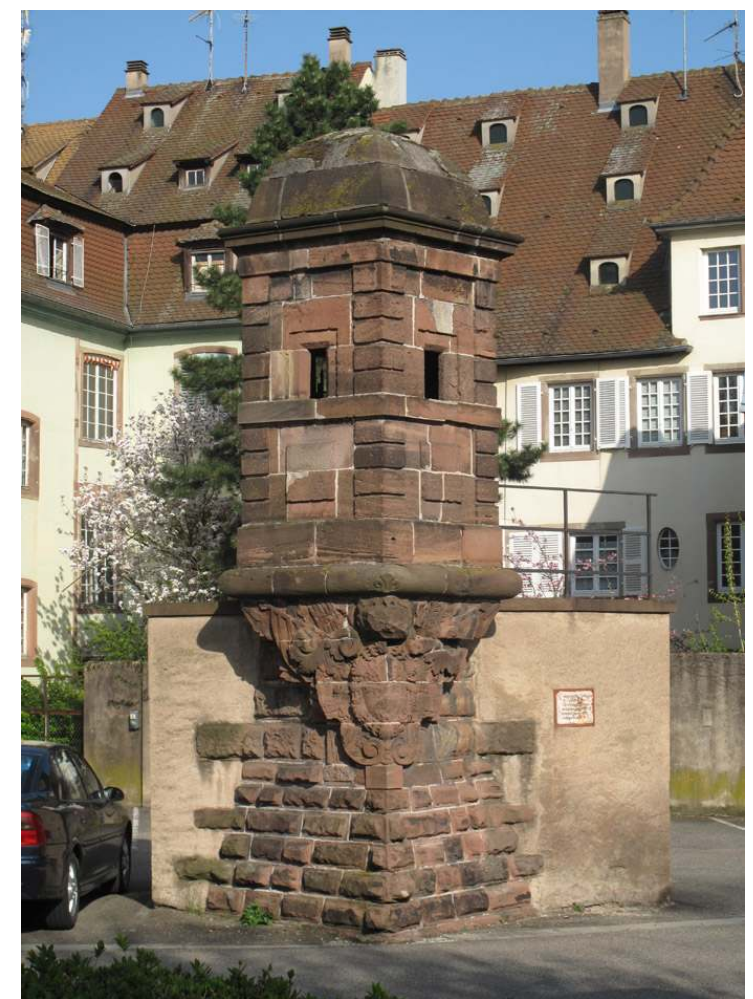

Échauguette, déplacée par les Allemands, de l'ancienne demi-lune située devant la porte SainteÉlisabeth. Phot. M.-D. Waton, 2010.

(c) DRAC Alsace, SRA.

\section{Politique patrimoniale des éléments défensifs et leur insertion dans des aménagements contemporains}

43 La destruction du système bastionné, à partir des années 1908-1909, a abondamment été photographiée (fig. $\mathbf{n}^{\circ} \mathbf{2 5}$ ). Les archives (Denkmalarchiv), en cours de numérisation, sont conservées par la CRMH de la DRAC Alsace. 


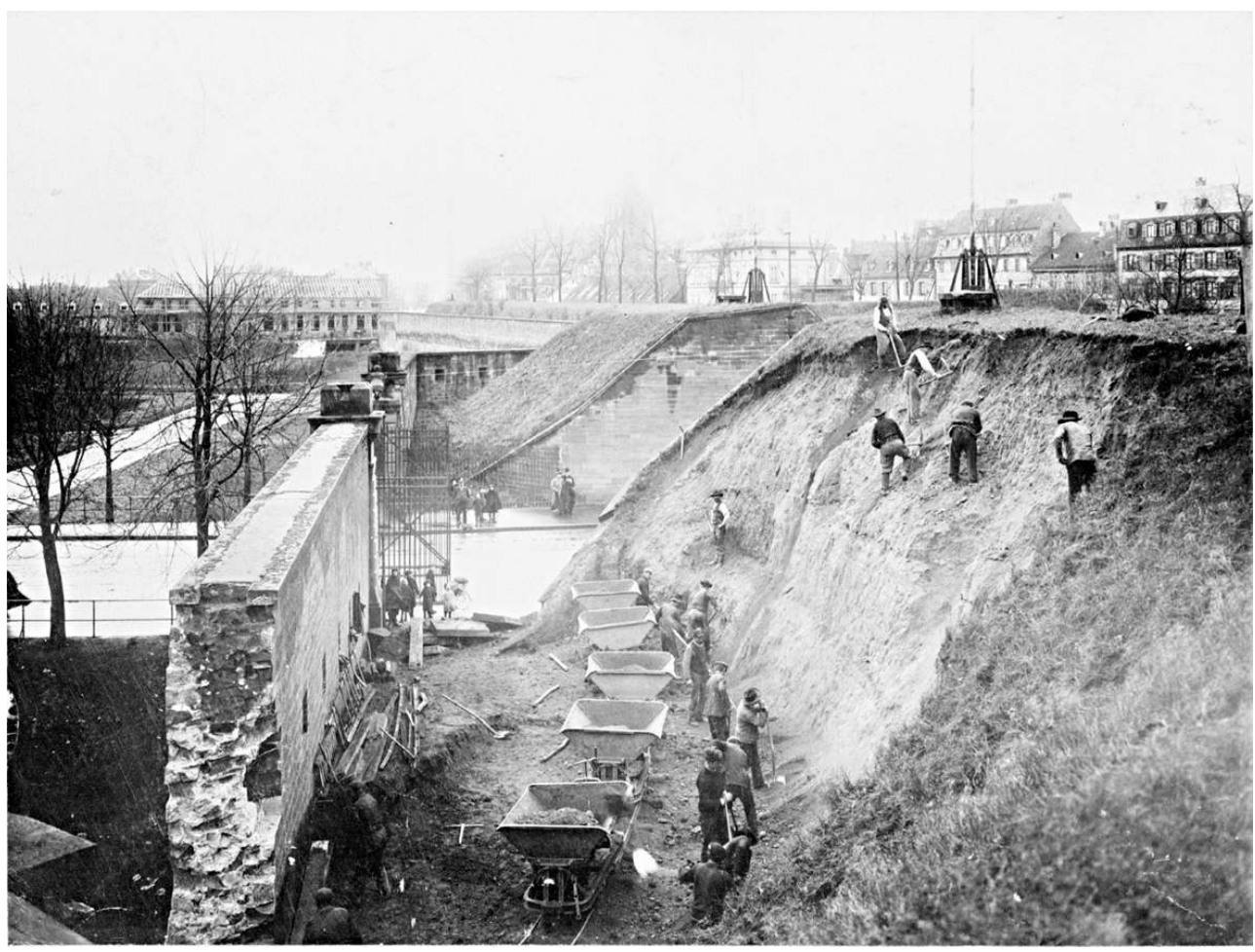

Le système bastionné sud en cours de démolition.

(c) DRAC Alsace, CRMH, ICO482C003_022_nuc2, [1908].

44 Si certains éléments défensifs ont été restaurés, ainsi la Spitalthor dans les années 1990, ou partiellement conservés dans des ouvrages protégés, comme les fondations romaines dégagées au sein du Grenier d'Abondance, largement réaménagé dans les années 2000, la restauration actuelle des bastions avancés des Ponts-Couverts et du bastion SaintMaximilien n'a pas pu être accompagnée par les archéologues, au contraire de celle à venir relative au barrage Vauban.

Lors de constructions nouvelles, des éléments du système défensif ont été maintenus; la portion d'escarpe entre la Spitalthor et la tour pentagonale, place de l'Hôpital, attribuée au milieu-seconde moitié du XIV ${ }^{e}$ siècle d'après la typologie des blocs à bossage, forme l'un des murs visibles du parking souterrain tout comme celle qui avait été reconnue au $\mathrm{n}^{\circ} 9$ de la rue des Glacières sur $70 \mathrm{~m}$. Une partie du tronçon épiscopal, quoique remaniée ${ }^{40}$, est visible aux anciens Haras, dont la protection au titre des Monuments historiques ne concerne que les éléments du XVIII ${ }^{e}$ siècle ${ }^{41}$. Dans l'immeuble qui surplombe l'enceinte, à l'angle des rues des Glacières et Sainte-Élisabeth, sont reconnaissables quelques vestiges de défense liés à la Porte Sainte-Élisabeth.

Quai de Turckheim, d'après les rapides observations effectuées après le décrépissage de l'enceinte au nord de la tour classée et qui semblent indiquer une absence de chaînage entre ces deux éléments, le mur défensif avait anciennement été intégré dans l'habitat et utilisé comme façade ; aucun percement nouveau n'a été autorisé.

Quant aux fortifications allemandes dont le dispositif fut déclassé après 1918, certains éléments furent détruits tandis qu'une ceinture verte était constituée sur son emprise, et des équipements aménagés (terrain de sport); le glacis a été remplacé par une voie 
rapide. Sur la partie conservée a été aménagé un parc (Parc du Glacis), tandis qu'après réhabilitation des bâtiments, plusieurs bastions sont de nouveau utilisés: services, gendarmerie nationale, ateliers d'artistes. L'ouverture de la porte dite Kriegstor permet de relier par une piste cyclable les quartiers de la gare et de Cronenbourg, séparés par la voie ferrée et la rocade de Strasbourg. Ce patrimoine reste, néanmoins, encore peu connu des circuits touristiques, voire de beaucoup de Strasbourgeois même.

En ce qui concerne les protections patrimoniales qui ont été prises à l'égard des enceintes de Strasbourg, on peut constater que les quelques éléments retrouvés anciennement lors de travaux ont été conservés par les Allemands et que la majorité des éléments en élévation ont été protégés au titre des Monuments historiques dans l'entre deux-guerres. Par la suite, la protection ou la conservation ont parfois porté sur des vestiges retrouvés lors de fouilles archéologiques, la plus grande partie des fortifications médiévales ou modernes étant simplement arasée. Actuellement, avec le dernier arrêté relatif aux fortifications allemandes, on s'est à nouveau tourné vers des éléments en usage, encore hors du sol et d'époque plus récente.

Quelques tronçons de l'extension des fortifications au sud de Strasbourg, visible sur un plan de 1725 (fig. $\mathbf{n}^{\circ} \mathbf{2 6}$ ), encore conservés dans le quartier du Neuhof, mériteraient une protection.

Figure 26

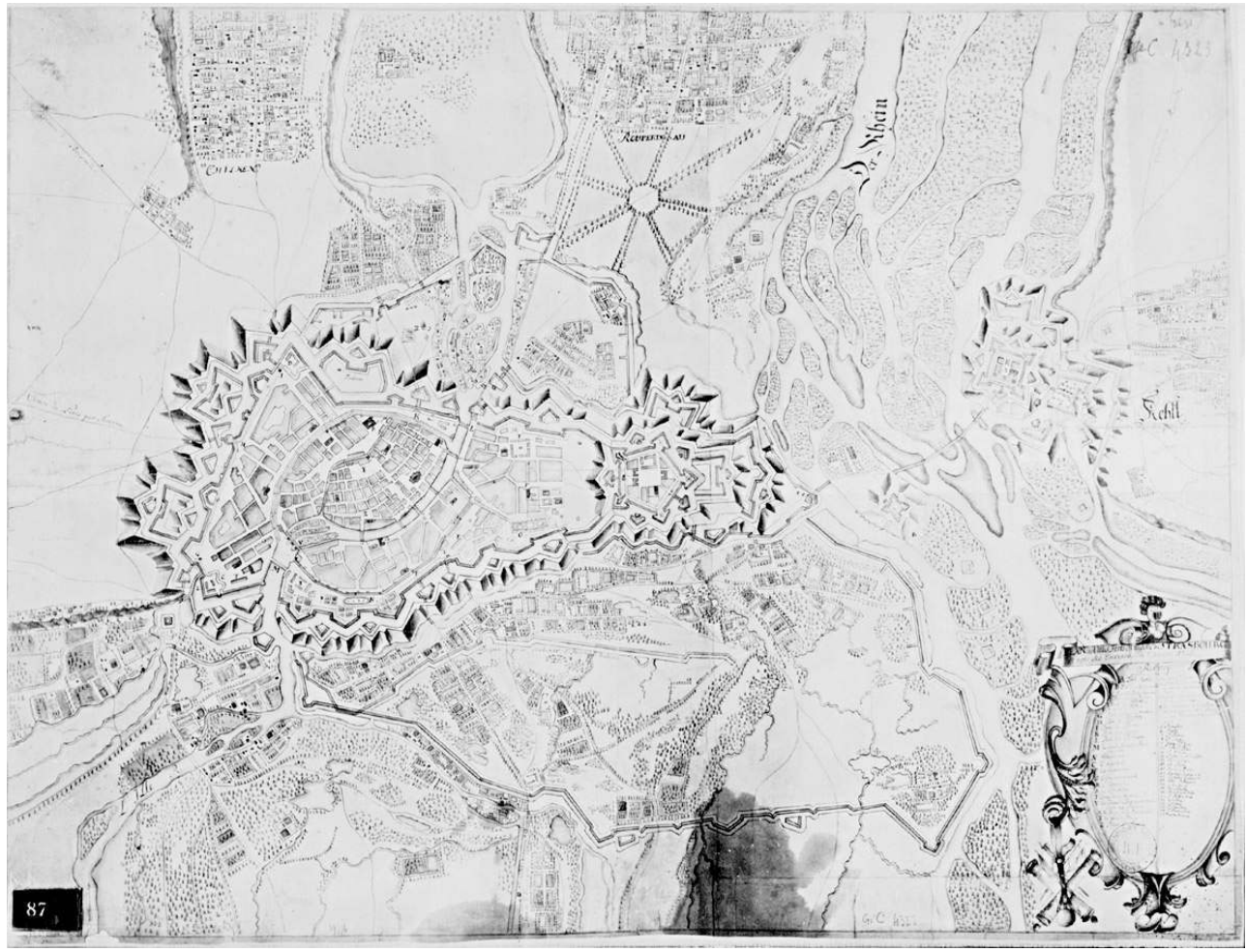

Plan des fortifications de Strasbourg et ses environs (1725).

(c) DRAC Alsace, CRMH, ICO482D001_003_nuc2.

50 C'est ainsi que, de quadrangulaire puis ovale et ensuite piriforme, la surface fortifiée ${ }^{42}$ s'est progressivement accrue en s'étirant d'est en ouest pour atteindre une forme globalement elliptique à la fin du XIX ${ }^{e}$ siècle. L'enceinte du Bas-Empire a perduré quelque neuf siècles et son front nord-est, dix-sept ; trois tours seulement sont conservées dont 
deux sont protégées au titre des Monuments historiques. Seules des traces parcellaires témoignent du premier Moyen Âge. Quant aux fortifications du second Moyen Âge ${ }^{43}$, quelques tours et tours-portes ont été protégées autour des années 30, ce qui est peu au regard de leur nombre. Avec les courtines, elles sont, pour la plupart, enfouies dans le sous-sol de Strasbourg tout comme le sont les vestiges de l'époque moderne, dont seuls deux monuments ont connu, jusqu'à présent, une protection. Tout récemment, on s'est intéressé à la période d'occupation allemande.

\section{NOTES}

1. - Contrairement à ce que propose Gertrud Kunhle qui s'appuie sur un schéma théorique, sans prendre en compte les données de fouille antérieures et les indices parcellaires livrés par la ville actuellement: KUNHLE, G. Des données nouvelles sur le camp légionnaire d'Argentorate. In: SCHNITZLER, B. et KUNHLE, G. (dir.). Strasbourg - Argentorate : un camp légionnaire sur le Rhin ( ${ }^{\mathrm{er}}$ au IV $\mathrm{IV}^{\mathrm{e}}$ siècle après J.-C.). Catalogue d'exposition. Strasbourg: Musées de la Ville de Strasbourg, 2010, p. 42-44 (fouilles récentes ; 8).

2. - Mise au jour sur le site d'Istra, rue des Juifs. Voir : WATON, M.-D. Problèmes de l'implantation et de l'occupation du camp romain : l'exemple d'Istra. In : SCHNITZLER, Bernadette (dir.) - 12. Aux origines de Strasbourg. Catalogue d'exposition. Strasbourg: Musées de la Ville de Strasbourg, 1988, p. 46-51. Initialement, la porta praetoria se trouvait à l'est, face à l'ennemi.

3. - Voir: BAUDOUX, Juliette, FLOTTÉ, Pascal, FUCHS, Matthieu, WATON, M.-D. Carte archéologique de la Gaule, Strasbourg, 67/2. Paris: Académie des Inscriptions et Belles Lettres, 2002, p. 83.

4. - Ce « chemin creux », transformé ultérieurement en fossé (cf. infra) a été mis au jour par Richard Nilles, lors des investigations archéologiques liées à la réalisation du tramway ligne $\mathrm{B}$ et dirigées par Juliette Baudoux, en 1998. Voir BAUDOUX, J. Strasbourg : Ligne B du tramway. Bilan Scientifique de la Région Alsace 1999. MCC : SRA, 2003, p. 50-51.

5. - NILLES, R. Strasbourg: Rue du Vieil Hôpital. Bilan Scientifique de la Région Alsace 2003. MCC : SRA, 2008, p. 43.

6. - Fouille AFAN 1990 : Ours Blanc, responsable d'opération Christian Peter; cette découverte pourrait conforter la version du chroniqueur J. T. de Koenigshoven, selon laquelle une première extension aurait été réalisée au temps du duc Adalbert vers 700.

7. - BAUDOUX, J., NILLES, R. Découverte d'un important fossé défensif d'époque médiévale, rue de Molsheim à Strasbourg. Cahiers alsaciens d'Archéologie, d'Art et d'Histoire, 1999, XLII, p. 65-77.

8. - WATON, M.-D., DORMOY, Chr. Strasbourg (Bas-Rhin), 4 rue des Veaux : l'évolution d'une berge médiévale de l'an mil au XIII ${ }^{\mathrm{e}}$ siècle. Cahiers alsaciens d'Archéologie, d'Art et d'Histoire, 1999, XLII, p. 79-115 ; fouille inédite PAIR 2009 : 12-16 rue des Veaux, responsable d'opération Maxime Werlé.

9. - DOLLINGER, P. Le premier statut municipal de Strasbourg (XII ${ }^{\mathrm{e}}$ siècle). Annuaire de la Société des Amis du Vieux Strasbourg, 1972-1973, 3, p. 13-35. Il y est question de deux types de fossés se développant dans la partie ouest de l'ellipse.

10. - HENIGFELD, Yves, CICUTTA, Heidi, PUTELAT, Olivier, SCHNEIDER, Nathalie. Strasbourg, Boulevard Wilson (Bas-Rhin). Rapport final d'opération. INRAP : SRA, 2008. 
11. - À l'emplacement du fossé antérieurement réalisé, l'arrière du mur a été comblé avec les graviers issus du fossé nouvellement aménagé en avant de la structure maçonnée, formant ainsi un excellent drain pour cette dernière, en présentant une stratigraphie inversée.

12. - Voir: SCHWIEN, Jean-Jacques. Strasbourg. Document d'évaluation du patrimoine archéologique urbain. Centre National d'Archéologie Urbaine : Tours, 1992, p. 3, 126.

13. - WERLÉ, M. Strasbourg: 16 rue Prechter. Bilan Scientifique de la Région Alsace 2000. MCC : SRA, 2005, p. 38.

14. - Pour le tracé de cette enceinte, un diagnostic a permis de préciser sa localisation dans les murs du Lycée Jean Rostand : HENIGFELD, Y. Strasbourg : Lycée Jean Rostand. Bilan Scientifique de la Région Alsace 2004. MCC : SRA, 2010, p. 36-38.

15. - NILLES, R. Strasbourg: Rue des Glacières. Bilan Scientifique de la Région Alsace 1997. MCC : SRA, 1999, p. 29.

16. - Nous renvoyons au site suivant qui complète les informations de la base Mérimée : http:// www.monuments-alsace.com/classement/bas-rhin/strasbourg-fortifications/index.html\#un.

17. - Voir dans la base Mérimée : notice PA00085123.

18. - KUHNLE, G. Strasbourg: Grenier d'Abondance. Bilan Scientifique de la Région Alsace 1999. MCC : SRA, 2003, p. 53. Voir dans la base Mérimée : notice PA67000028.

19. - Voir dans la base Mérimée : notice PA00085033.

20. - Voir dans la base Mérimée : notice PA00085191.

21. - WERLÉ, M. Strasbourg: 9/10, place de l'Hôpital. Bilan Scientifique de la Région Alsace 1999. MCC : SRA, 2003, p. 60.

22. - WATON, M.-D., WERLÉ, M., MUNIER, C.. L'environnement défensif singulier de la place de l'Hôpital à Strasbourg. Cahiers alsaciens d'Archéologie, d'Art et d'Histoire, 2000, XLIII, p. 75-77.

23. - Voir dans la base Mérimée : notice PA00085035.

24. - Voir dans la base Mérimée : notice APMH0107387.

25. - Voir dans la base Mérimée : notice PA00085192.

26. - HENIGFELD, Y. Strasbourg: Angle du boulevard Wilson et de la rue Wodli. Bilan Scientifique de la Région Alsace 1999. MCC: SRA, 2003, p. 52 ; WATON, M.-D. Strasbourg: Boulevard Wilson - Rue Wodli. Bilan Scientifique de la Région Alsace 2000. MCC : SRA, 2005, p. 34 .

27. - Voir dans la base Mérimée : notice PA67000051.

28. - Voir dans la base Mérimée : notice PA00085194.

29. - Les différents plans réalisés par D. Specklin entre 1577 et 1589 ont constitué une source admirablement exploitée par F. von Apell, dans son ouvrage paru en 1902 sur Strasbourg : APELL, F. von. Geschichte der Befestigung von Strassburg in Elsass vom Wiederaufbau der Stadt nach der Völkerwanderund bis zum Jahre 1681. Strasbourg, 1902.

30. - Les minutes élaborées pour sa réalisation sont très précises et permettent d'affiner les plans. Nous renvoyons au site suivant pour une première approche des plans successifs de Strasbourg : http://archives.ac-strasbourg.fr/microsites/hist_geo01/sig-stg.

31. - WATON, M.-D. Strasbourg: Futur Musée d'Art Moderne et Contemporain. Bilan Scientifique de la Région Alsace 1993. MCC : SRA, 1995, p. 38.

32. - Voir dans la base Mérimée : notice PA00085039.

33. - Voir dans la base Mérimée : notice PA00085019.

34. - BURTSCHER, Ph., HOFF, F. Les fortifications allemandes d'Alsace-Lorraine, 1870-1918. Histoire et Collections : Paris, 2008.

35. - Voir dans la base Mérimée : notice PA67000083.

36. - GARSCHA, F. Die Frellegung des mittelalterlichen Kornspeichers über der römischen Mauer in Strassburg. Volk und Vorzeit, 1941, p. 33-46. 
37. - WATON, M.-D. Parking Saint-Aurélie. Bilan Scientifique de la Région Alsace 1992. MCC : SRA, 1994, p. 34 ; BAUDOUX, J. Strasbourg, 1 boulevard de Metz. Bilan Scientifique de la Région Alsace 2004. MCC : SRA, 2010, p. 36.

38. - Sur ce chantier, deux tourelles avaient été rencontrées et le mur, dégagé sur près de $200 \mathrm{~m}$ : KERN, E. Enceinte du « 3e agrandissement » de la ville, construite entre 1374 et 1390. In : Collectif. Vivre au Moyen Âge : 30 ans d'archéologie médiévale en Alsace. Strasbourg : Les Musées de la Ville de Strasbourg, 1990, p. 112-113 (plan).

39. - KOCH, Jacky. Strasbourg: Hôpital civil - Pôle cœur-poumon. Bilan Scientifique de la Région Alsace 1999. MCC : SRA, 2003, p. 52-53.

40. - Observations 2001. In : NILLES, R. Strasbourg, Établissement des Diaconesses, 2, rue Ste Élisabeth, rapport de fouille d'évaluation archéologique du 14/02 au 22/02/2001. AFAN : SRA, 2001 et $\mathrm{KOCH}$, J. Strasbourg (Alsace, Bas-Rhin) - Haras. Rapport de diagnostic. PAIR : SRA, 2010.

41. - Voir dans la base Mérimée : notice PA00085042.

42. - LIVET, Georges, RAPP, Francis (dir.). Histoire de Strasbourg des origines à nos jours. Strasbourg: Dernières Nouvelles d'Alsace, 1980-1982; LIVET, G., RAPP, F. dir. Histoire de Strasbourg. Toulouse : Privat, 1987 ; SCHWIEN, J.-J., HENIGFELD, Y., KELLER, M., WATON, M.-D., ZUMSTEIN, H. Histoire et archéologie des enceintes de Strasbourg. $\mathbf{1 2 1}^{\mathrm{e}}$ Congr. nat. soc. hist. scient. Archéologie - Enceintes Urbaines. Nice, 1996, p. 135-162 ; HENIGFELD, Y., SCHWIEN, J.J., WERLÉ, M., SEILLER, M. L'apport de l'archéologie à la connaissance de la ville médiévale : le cas de Strasbourg. In: CHAPELOT, J. (dir.). Trente ans d'archéologie médiévale en France : un bilan pour un avenir. CRAHM : Caen, 2010, p. 351-368.

43. - APELL, F. von. Geschichte der Befestigung von Strassburg in Elsass vom Wiederaufbau der Stadt nach der Völkerwanderund bis zum Jahre 1681. Strasbourg, 1902 ; WATON, M.-D. Strasbourg, image d'une ville en 1400 : Les fortifications. In : Collectif. - 1400, l'Alsace dans l'Europe gothique. Lyon : Lieux Dits, 2008, p. 44-51; WERLÉ, M. L'enceinte et ses composantes : 2. En Alsace. In : HENIGFELD, Y. et MASQUILIER, A. (dir.). Archéologie des enceintes urbaines et de leurs abords en Lorraine et en Alsace (XII ${ }^{\mathrm{e}}-\mathrm{XV}^{\mathrm{e}}$ siècle). Revue archéologique de l'Est, 26e suppl. Dijon, 2008, p. 414-435.

\section{RÉSUMÉS}

Les Strasbourgeois n'ont pris conscience de la richesse de leur patrimoine militaire qu'au cours $\mathrm{du} \mathrm{XX}^{\mathrm{e}}$ siècle. Deux tours de l'enceinte du Bas-Empire ont été protégées au titre des Monuments historiques. Aucun des vestiges du premier Moyen Âge ne l'est : des traces parcellaires attestent cette période. Quant aux fortifications du second Moyen Âge, quelques tours ont été protégées dans les années 30, ce qui est peu au regard de leur nombre. Elles sont enfouies dans le sous-sol de Strasbourg comme les vestiges de l'époque moderne, dont deux monuments phares ont fait l'objet d'une protection. Des éléments de l'époque allemande ont tout récemment été protégés.

The inhabitants of Strasbourg became aware of the wealth of their military heritage only during the twentieth century. Two towers of the surrounding wall of the late Empire Roman town were protected according to the legislation on historic monuments. None of the remains from the early Middle Ages are protected, although land use patterns give information on that period. As for the fortifications of the later Middle Ages, some towers were protected in the 1930s, although 
only few considering their number. They are buried in the underground levels of the city like vestiges of the beginnings of the modern period, for which two major monuments have been protected. Elements of the German period between 1870 and 1918 have been protected only recently.

INDEX

Keywords : antique, City wall, episcopal, gate, land plan, medieval, moat, modern, protection, Strasbourg, surrounding wall, tower

Mots-clés : antique, enceinte, épiscopal, fossé, médiéval, moderne, muraille, parcellaire, porte, protection, Strasbourg, tour

\section{AUTEUR}

\section{MARIE-DOMINIQUE WATON}

IE, DRAC d'Alsace, service régional de l'archéologie, avec la collaboration d'Olivier Kayser, conservateur régional de l'archéologie d'Alsace dominique.waton@culture.gouv.fr 